\title{
ON QUASI-F-ORTHODOX SEMIGROUPS
}

\author{
by BERND BILLHARDT and MÁRIA B. SZENDREI*
}

(Received 28th September 1993)

Dedicated to the memory of A. H. Clifford

\begin{abstract}
An orthodox semigroup $S$ is termed quasi-F-orthodox if the greatest inverse semigroup homomorphic image of $S^{1}$ is $F$-inverse. In this paper we show that each quasi- $F$-orthodox semigroup is embeddable into a semidirect product of a band by a group. Furthermore, we present a subclass in the class of quasi- $F$-orthodox semigroups whose members $S$ are embeddable into a semidirect product of a band $B$ by a group in such a way that $B$ belongs to the band variety generated by the band of idempotents in $S$. In particular, this subclass contains the $F$-orthodox semigroups and the idempotent pure homomorphic images of the bifree orthodox semigroups.
\end{abstract}

1991 Mathematics subject classification (Amer. Math. Soc): 20M10, 20M19.

\section{Introduction}

The structure of $E$-unitary inverse semigroups is described in McAlister's $P$-theorem (cf. [10, VII.1.14]) by means of partially ordered sets, semilattices and groups. By applying the $P$-theorem, $O^{\prime} C$ arroll [7] proved that each $E$-unitary inverse semigroup is embeddable into a semidirect product of a semilattice by a group. On the other hand, it is easy to obtain a $P$-representation of an $E$-unitary inverse semigroup $S$ provided $S$ is embedded into a semidirect product of semilattice by a group. This idea led the second author in [13] to try to generalize McAlister's $P$-theorem for $E$-unitary regular semigroups by investigating whether each $E$-unitary regular semigroup is embeddable into a semidirect product of a band by a group. Notice that a semidirect product of a band by a group, and so each regular subsemigroup in it is necessarily $E$-unitary.

For brevity, we will say that an $E$-unitary regular semigroup $S$ is embeddable if it is embeddable into a semidirect product of a band $B$ by a group. If $B$ can be chosen from the band variety generated by the band of idempotents in $S$ then $S$ is called strictly embeddable. A class of E-unitary regular semigroups is termed embeddable [strictly embeddable] if each member in it is embeddable [strictly embeddable].

So far, two embeddable classes of $E$-unitary regular semigroups has been known,

*The results of the paper were mainly obtained while the second author was a Humboldt fellow at the FB Mathematik, AG 1, Technische Hochschule Darmstadt. The excellent circumstances provided by the fellowship and by the THD are gratefully acknowledged. Research partially supported by Hungarian National Foundation for Scientific Research grant no. 1903. Most results of the paper were announced at the International Conference on Semigroups, Luino, 22-27 June, 1992. 
namely, that of all $E$-unitary regular semigroups with regular band of idempotents (cf. [13]) and that of all idempotent pure homomorphic images of the bifree orthodox semigroups (cf. [14]). In fact, these classes are also strictly embeddable. The aim of this paper is to present a new embeddable class and a strictly embeddable subclass in it.

An $F$-inverse semigroup is an inverse semigroup whose congruence classes modulo the least group congruence contain greatest elements with respect to the natural partial order. The structure of $F$-inverse semigroups which are easily seen to be $E$-unitary monoids is well known (cf. [10, VII.6.9]). Regular semigroups satisfying the same condition were investigated by Edwards [1]. They turned out to be orthodox, what is more, $E$-unitary regular monoids, therefore she called them $F$-orthodox semigroups. Here we introduce a larger class. By a quasi-F-orthodox semigroup we mean an orthodox semigroup $S$ such that the greatest inverse semigroup homomorphic image of $S^{1}$ is $F$-inverse. Moreover, we introduce a subclass in the class of quasi- $F$-orthodox semigroups whose members are called generalized F-orthodox semigroups. It contains all $F$-orthodox semigroups and all idempotent pure homomorphic images of the bifree orthodox semigroups.

In the main result of the paper we prove that the quasi- $F$-orthodox semigroups are embeddable and the generalized $F$-orthodox semigroups are strictly embeddable. The latter result provides us with a new, purely algebraic proof for the main result in [14].

\section{Preliminaries}

In this section we summarize the notions and results needed in the paper. For the undefined notions and notations the reader is referred to [4].

The equality relation on a set $A$ is denoted by id $A$ or, briefly, by id.

If $A$ is a non-empty set then $A^{+}$and $\left(A^{+}\right)^{1}$ stands for the free semigroup and for the free monoid on $A$, respectively.

If $\phi: S \rightarrow T$ is a homomorphism then the congruence on $S$ induced by $\phi$ is denoted by $\equiv_{\phi}$.

Given a regular semigroup $S$, its set of idempotents is denoted by $E_{S}$ or, if it causes no confusion, then simply by $E$. The identity element of a monoid, in particular, of a group is denoted by 1 .

We will use the notation $\leqq_{\mathscr{R}}$ for the quasi-order relation defined on a semigroup $S$ by means of principal right ideals. Namely, we define $a \leqq_{\mathscr{R}} b$ for some $a, b \in S$ if $a S^{1} \subseteq b S^{1}$. Obviously, we have $\leqq \mathscr{R} \geqq \geqq_{\mathscr{R}}=\mathscr{R}$. If we want to indicate that Green's relation $\mathscr{R}$ is considered on semigroup $S$ then we will write $\mathscr{R}_{S}$.

The following useful property of relation $\mathscr{R}$ on regular semigroups was proved in [3].

Result 1.1. Let $S$ be a regular semigroup and $\theta$ a congruence on it. If $\bar{s}, \bar{t} \in S / \theta$ such that $\bar{s} \mathscr{R}_{S / \theta} t$ then there exist $s \in \vec{s}$ and $t \in \bar{t}$ such that $s \mathscr{R}_{S} t$.

Let $S$ be a regular semigroup. As usual, $\gamma$ stands for the least inverse semigroup congruence on $S$ and $\sigma$ for the least group congruence on $S$. The greatest factor group $S / \sigma$ of $S$ will be denoted by $G_{S}$ or, simply, by $G$. 
Let $\theta$ be a congruence on $S$. Then, as usual, we denote its kernel $\{s \in S: s \theta e$ for some $e \in E\}$ by $\operatorname{ker} \theta$ and its $\operatorname{trace} \theta \mid E$ by $\operatorname{tr} \theta$. Obviously, we have $E \subseteq \operatorname{ker} \theta$. It is well known that if $S$ is orthodox then $\operatorname{ker} \theta$ is an orthodox subsemigroup in $S$. On the other hand, if $\theta$ is a group congruence then $\operatorname{ker} \theta=\{s \in S: s \theta=1\}$ and $\operatorname{ker} \theta$ is a regular subsemigroup in $S$.

We will need to consider $(S / \theta)^{1}$ as a factor semigroup of $S^{1}$. It is easy to check that $(S / \theta)^{1}$ is isomorphic to $S^{1} / \theta^{1}$ where

$$
\theta^{1}=\theta \cup\{(s, 1),(1, s): s \in S \text { such that } s \theta \text { is an identity in } S / \theta\} \cup(1,1) .
$$

We will identify $(S / \theta)^{1}$ with $S^{1} / \theta^{1}$ under this isomorphism. Furthermore, we notice that if $\theta \subseteq \sigma$ then $\theta^{1} \subseteq \sigma^{1}, \sigma / \theta$ and $\sigma^{1} / \theta^{1}$ are the least group congruences on $S / \theta$ and $S^{1} / \theta^{1}$, respectively, and $G_{S / \theta}$ is isomorphic to $G_{S^{1} / \theta_{1}}$.

A regular semigroup is called $E$-unitary if $E$ is a (left and/or right) unitary subset in $S$. A regular semigroup is easily seen to be $E$-unitary if and only if $\operatorname{ker} \sigma=E$, and hence an $E$-unitary regular semigroup is necessarily orthodox.

We will need the following property of $E$-unitary regular semigroups (cf. [13]).

Result 1.2. In every E-unitary regular semigroup, we have $\mathscr{H} \cap \sigma=\mathrm{id}$.

The natural partial order on an inverse semigroup $S$ is defined in the following way: for any $s, t \in S$, we say that $s \leqq t$ if $s=e t$ for some $e \in E$. The following is well known (cf. [10, II.1.6]):

Result 1.3. In an inverse semigroup $S$, we have $s \leqq t$ for some $s, t \in S$ if and only if $s=s t^{-1} s$.

The notion of the natural partial order was generalized by Nambooripad [6] for regular semigroups as follows: if $S$ is a regular semigroup and $s, t \in S$ then we define $s \leqq t$ if $s \leqq t$ and $s=e t$ for some $e \in E \cap R_{s}$. The relation $\leqq$ is shown to be self-dual. It is easy to see that if $S$ is an inverse semigroup then the partial order defined in this way coincides with the former one. Moreover, the following assertion is also straighforward from the definition.

Result 1.4. Let $S$ be a regular semigroup and $\theta$ a congruence on it. If $s, t \in S$ such that $s \leqq t$ then $s \theta \leqq t \theta$ in $S / \theta$.

A band is termed left normal [left regular] if it satisfies the identity $a b c=a c b$ $[a b a=a b]$. Recall from Petrich [9] the following properties of such bands.

Result 1.5. (i) $A$ band $B$ is left normal if and only if $a B$ is a semilattice for every $a \in B$.

(ii) $A$ band is left regular if and only if $\mathscr{R}=\mathrm{id}$ in it. 
A semigroup is called $\mathscr{R}$-unipotent [ $\mathscr{L}$-unipotent] if each $\mathscr{R}$-class [ $\mathscr{L}$-class] contains a unique idempotent. The following characterization is from Venkatesan [18].

Result 1.6. The following two conditions are equivalent for a semigroup $S$.

(i) $S$ is $\mathscr{R}$-unipotent.

(ii) $S$ is an orthodox semigroup whose band of idempotents is left regular.

Given a regular semigroup $S$, it is standard to verify that there exists a least $\mathscr{R}$-unipotent [ $\mathscr{L}$-unipotent] congruence on $S$. We will denote it by $\alpha_{S}\left[\beta_{S}\right]$ or, simply, by $\alpha[\beta]$. It is straightforward that $\alpha, \beta \subseteq \gamma \subseteq \sigma$.

As far as the $E$-unitary $\mathscr{R}$-unipotent semigroups is concerned, we need the following property from Takizawa [16].

Result 1.7. In every E-unitary $\mathscr{R}$-unipotent semigroup, we have $\mathscr{R} \cap \sigma=\mathrm{id}$.

Now we recall the basic notions on graphs and semigroupoids needed later. We follow the terminology in Tilson [17].

A graph $X$ consists of a set of objects denoted by $\operatorname{Obj}(X)$ and, for every pair $i, j \in \operatorname{Obj}(X)$, a set of arrows from $i$ to $j$ which is denoted by $X(i, j)$ and is called a hom-set. The different hom-sets are supposed to be disjoint. If $a \in X(i, j)$ then we also write that $\alpha(a)=i$ and $\omega(a)=j$. The set of all arrows will be denoted by $\operatorname{Arr}(X)$. The arrows $a, b$ are called coterminal if $a, b \in X(i, j)$ for some $i, j \in \operatorname{Obj}(X)$ and are termed consecutive provided $\omega(a)=\alpha(b)$.

By a subgraph of a graph $X$ we mean a graph $Y$ such that $\operatorname{Obj}(Y) \subseteq \operatorname{Obj}(X)$ and $Y(i, j) \subseteq X(i, j)$ for every $i, j \in \operatorname{Obj}(Y)$.

A semigroupoid is a graph $C$ equipped with a composition which assigns to every pair of consecutive arrows $a \in C(i, j), b \in C(j, k)$ an arrow $a b \in C(i, k)$ such that the composition is associative, that is, for any arrows $a \in C(i, j), b \in C(j, k)$ and $c \in C(k, l)$, we have $(a b) c=a(b c)$. If, furthermore, for every $i \in \operatorname{Obj}(C)$, we have an element $1_{i} \in C(i, i)$ such that

$$
\begin{aligned}
& 1_{i} a=a \text { and } \quad b 1_{i}=b \quad \text { for every } a, b \in \operatorname{Arr}(C) \\
& \text { with } \alpha(a)=i \text { and } \omega(b)=i,
\end{aligned}
$$

then we term $C$ a category.

Observe that $C(i, i)$ is either empty or a semigroup for each $i \in \operatorname{Obj}(C)$. For the sake of uniformity, we will consider the empty set also as a semigroup, and we term $C(i, i)$ the local semigroup of $C$ at $i$.

If $C$ is a category then $1_{i}$ is the identity in the local semigroup at $i$. If $C$ is a semigroupoid then, for every $i \in \operatorname{Obj}(C)$, we can adjoin a new element $1_{i}$ to $C(i, i)$ provided it has no element satisfying (1.1). Thus we obtain a category which we denote by $C^{1}$. 
In particular, a set $A$ can be considered as a graph with one object whose unique hom-set is $A$ and, similarly, a semigroup $S$ can be considered as a semigroupoid with one object whose unique hom-set is $S$.

Let $X, Y$ be two graphs. A graph function $f: X \rightarrow Y$ consists of an object function $f: \operatorname{Obj}(X) \rightarrow \operatorname{Obj}(Y)$ and, for every, $i, j \in \operatorname{Obj}(X)$, a hom-set function $f: X(i, j) \rightarrow Y(i f, j f)$. If $C, D$ are semigroupoids then by morphism of semigroupoids $\phi: C \rightarrow D$ we mean a graph function $\phi$ such that $a \phi \cdot b \phi=(a b) \phi$ for every pair $a, b$ of consecutive arrows in $C$. If the hom-set functions are injective then $\phi$ is termed a faithful morphism. If the object function is bijective and the hom-set functions are surjective then $\phi$ is said to be a quotient morphism. If both the object and the hom-set functions are bijective then $\phi$ is called an isomorphism.

By a congruence $\gamma$ on a semigroupoid $C$ we mean a family

$$
\gamma=\{\gamma(i, j): i, j \in \operatorname{Obj}(C)\}
$$

of equivalence relations $\gamma(i, j)$ on $C(i, j)$ such that, for every $a \in C(i, j), b, c \in C(j, k)$ and $d \in C(k, l)(i, j, k, l \in \mathrm{Obj}(C))$, the relation $b \gamma(j, k) c$ implies $a b \gamma(i, k) a c$ and $b d \gamma(j, l) c d$. For simplicity, we will often write $\gamma$ instead of $\gamma(i, j)$. Note that $\gamma(i, i)$ is a congruence on the local semigroup $C(i, i)$. If $a \in C(i, j)$ then the equivalence class containing $a$ will be denoted by $a \gamma(i, j)$ or, simply, by $a \gamma$.

Given a congruence $\gamma$ on a semigroupoid $C$, we can define the quotient semigroupoid $C / \gamma$ as follows: $\operatorname{Obj}(C / \gamma)=\operatorname{Obj}(C),(C / \gamma)(i, j)$ is the set of all $\gamma(i, j)$-classes of $C(i, j)$ and the composition rule is given by

$$
\alpha \gamma \cdot b \gamma=(a b) \gamma \quad(a \in C(i, j), b \in C(j, k)) .
$$

Obviously, the congruence $\gamma$ on $C$ determines a quotient morphism $\gamma^{\sharp}: C \rightarrow C / \gamma$ whose object function is identical and whose hom-set functions assign the respective $\gamma$-class to each arrow. Conversely, if $\phi: C \rightarrow D$ is a quotient morphism then the family of equivalence relations on the hom-sets in $C$ determined by the hom-set functions is a congruence on $C$ which we will denote by $\equiv_{\phi}$. Moreover, $\phi$ induces an isomorphism $t: C / \equiv_{\phi} \rightarrow D$ such that $\phi=\equiv \equiv_{\bar{\phi}} l$.

Let $X$ be a graph. A non-empty path in $X$ is a finite sequence of consecutive arrows in $X$. If $p=e_{1} e_{2} \ldots e_{n}(n \geqq 1)$ where $\alpha\left(e_{1}\right)=i$ and $\omega\left(e_{n}\right)=j$ then we say that $p$ is a non-empty $(i, j)$-path. If $p$ is a non-empty $(i, j)$-path and $q$ is a non-empty $(j, k)$-path for some $i, j, k \in \operatorname{Obj}(X)$ than their concatenation $p q$ is a non-empty $(i, k)$-path.

Given any graph $X$, we will consider the free semigroupoid on $X$ and denote it by $X^{+}$. It can be represented in the following way:

$$
\begin{gathered}
\operatorname{Obj}\left(X^{+}\right)=\operatorname{Obj}(X), \\
X^{+}(i, j)=\{p: p \text { is a non-empty }(i, j) \text {-path in } X\}
\end{gathered}
$$

and the composition is given by concatenation. If we add an empty path $1_{i}$ to $X^{+}(i, i)$ for 
every $i \in \operatorname{Obj}\left(X^{+}\right)$and extend the composition by putting $1_{i} p=p 1_{j}=p$ for each $(i, j)$-path $p$ then the free category on $X$ is obtained which will be denoted by $\left(X^{+}\right)^{1}$.

Notice that a non-empty path $p=e_{1} e_{2} \ldots e_{n}\left(n \geqq 1, e_{k} \in \operatorname{Arr}(X), 1 \leqq k \leqq n\right)$ in $X^{+}$spans a subgraph in the graph $X$ whose set of objects is $\left\{\alpha\left(e_{k}\right): 1 \leqq k \leqq n\right\} \cup\left\{\omega\left(e_{n}\right)\right\}$ and whose set of arrows is $\left\{e_{1}, \ldots, e_{n}\right\}$.

Let $X$ be any graph. Denote $\operatorname{Arr}(X)$ by $A$. Clearly, there is a natural morphism $\eta^{1}$ from $\left(X^{+}\right)^{1}$ to $\left(A^{+}\right)^{1}$ which maps all objects of $\left(X^{+}\right)^{1}$ to the single object of $\left(A^{+}\right)^{1}$ and which maps each path in $\left(X^{+}\right)^{1}$ to the corresponding word in $\left(A^{+}\right)^{1}$. In particular, $\eta^{1}$ maps each empty path to the empty word. For notational convenience, we will often denote the word $p \eta^{1}$ corresponding to the path $p$ also by $p$. Denote the restriction of $\eta^{1}$ to $X^{+}$and $A^{+}$by $\eta$. Obviously, both $\eta^{1}$ and $\eta$ are faithful morphisms.

We conclude this section with the notion and the main properties of a semidirect product of a band by a group and recall the embeddability criterion in [13].

Let $B$ be a band and $H$ a group. Suppose that $H$ acts on $B$ by automorphisms on the left, that is, for every $h \in H$, an automorphism of $B$ is given, denoted by $h: B \rightarrow B, b \mapsto h b$, such that $h(g b)=(h g) b$ holds for every $g, h \in H$ and $b \in B$. Briefly, we will say only that $H$ acts on $B$. The semidirect product $B * H$ is defined on the underlying set $B \times H$ by the multiplication

$$
(a, g)(b, h)=(a \cdot g b, g h) \quad(a, b \in B, g, h \in H) .
$$

The following properties of this semidirect product are straightforward.

Result 1.8. Let $B$ be $a$ band and $H$ a group acting on $B$.

(i) The semidirect product $B * H$ is an orthodox semigroup with $E_{B * H}=\{(b, 1): b \in B\}$, and $E_{B * H}$ is isomorphic to $B$.

(ii) The second projection $\pi_{2}: B * H \rightarrow H,(b, h) \mapsto h$ is a homomorphism of $B * H$ onto $H$ with $\operatorname{ker}\left(\equiv_{\pi_{2}}\right)=E_{B * H}$. Consequently, $\equiv_{\pi_{2}}=\sigma$ and $B * H$ is E-unitary.

We present here the embeddability criterion in [13] in a slightly modified form (cf. [15]).

Let $\mathbf{V}$ be a variety of bands and $S$ an $E$-unitary regular semigroup. First we define a graph $C=C_{S}$ as follows.

$$
\begin{gathered}
\operatorname{Obj}(C)=G, \\
C(g, h)=\{(g, s) \in G \times S: g \cdot s \sigma=h\} \quad(g, h \in G) .
\end{gathered}
$$

One can equip $C$ with the following multiplication: if $(g, s) \in C(g, h)$ and $(h, t) \in C(h, i)$ then

$$
(g, s) \circ(h, t)=(g, s t)
$$

Clearly, $(g, s t) \in C(g, i)$ and this multiplication is associative. Thus $C=(C ; \circ)$ constitutes a 
semigroupoid. It is also straightforward that the local semigroups in $C$ are isomorphic to $E=\operatorname{ker} \sigma$. Note that this semigroupoid is closely related to the derived semigroupoid of the homomorphism $\sigma^{*}: S \rightarrow G$, cf. [17].

It is easily seen that if $S$ has an identity then $C_{S}$ is a category. What is more, in general, $C_{S^{1}}$ is isomorphic to $C_{S^{1}}^{1}$. If $S$ has no identity then, $(g, 1)(g \in G)$ will be considered as an empty arrow. More precisely, for every $g \in G$, the arrow $(g, 1) \in C_{S^{1}}(g, g)$ will be identified with the empty path $1_{g}$ in $\left(C_{S^{1}}^{+}\right)^{1}$, and so, in fact, $\left(C_{S^{1}}^{+}\right)^{1}$ will be identified with $\left(C_{S}^{+}\right)^{1}$. In particular, this ensures that $(g, 1) \eta^{1}$ is the empty word in $\left(A^{+}\right)^{1}$ where $A=\operatorname{Arr}(C)$.

Consider the identity graph function $\imath$ on $C$. The unique extension $\tilde{\imath}: C^{+} \rightarrow C$ of $\imath$ to $C^{+}$is a quotient morphism. So it determines a congruence $\equiv_{i}$ on the semigroupoid $C^{+}$. Denote the image of $\equiv_{i}$ under $\eta$, namely, $\left\{(p \eta, q \eta):(p, q) \in \equiv_{i}\right\}$ by $v$. Consider the congruence $\tau_{\mathrm{v}}$ on $A^{+}$generated by $\varrho(\mathbf{V}, A) \cup v$ where $\varrho(\mathbf{V}, A)$ is the fully invariant congruence on $A^{+}$corresponding to the band variety $\mathbf{V}$.

The following statement is routine to verify.

Result 1.9. For any words $x, y$ in $A^{+}$, we have $x \tau_{v} y$ if and only if there exists a finite sequence of words $x=w_{0}, w_{1}, \ldots, w_{n}=y$ such that, for any $i(0 \leqq i<n)$, the word $w_{i+1}$ is obtained from $w_{i}$ by one of the following rules:

(S1) $w_{i} \varrho(\mathbf{V}, A) w_{i+1}$,

(S2) $w_{i}=u a b v, w_{i+1}=u c v$ for some $u, v \in\left(A^{+}\right)^{1}$ and $a, b, c \in A$ with $a \circ b=c$ in $C$,

(S2') $w_{i}=u c v, w_{i+1}=u a b v$ for some $u, v \in\left(A^{+}\right)^{1}$ and $a, b, c \in A$ with $a \circ b=c$ in $C$.

Remark 1.10. In particular, if $\mathbf{V}$ is the variety of all bands then (S1) can be substituted by the following two rules:

[S1] $w_{i}=u x^{2} v, w_{i+1}=u x v$ for some $u, v \in\left(A^{+}\right)^{1}$ and $x \in A^{+}$,

[S1'] $w_{i}=u x v, w_{i+1}=u x^{2} v$ for some $u, v \in\left(A^{+}\right)^{1}$ and $x \in A^{+}$.

The embeddability criterion in [13] is the following.

Result 1.11. Let $\mathrm{V}$ be a variety of bands and $S$ an E-unitary regular semigroup. Then $S$ is embeddable into a semidirect product of a member in $\mathbf{V}$ by a group if and only if sot and $(1, s) \tau_{\mathrm{v}}(1, t)$ imply $s=t$ for every $s, t \in S$.

\section{A result on extensions of completely regular semigroups by groups}

In this section we present the result which serves as a basis in the inductive proof of strict embeddabilty in Section 4. We apply this result in this paper only for band varieties but we decided to present it for any variety of completely regular semigroups since its proof is not more complicated in this generality, and, on the other hand, we consider it as an important tool in proving strict embeddability for regular semigroups being extensions of completely regular semigroups by groups.

First we recall several notions, notations and results in connection with varieties of completely regular semigroups due to Polák [11].

A completely regular semigroup is usually considered a unary semigroup where the 
unary operation assigns each element its inverse in the maximal subgroup containing it. It is well known that the class of all completely regular semigroups forms a variety of unary semigroups and it is defined by the following identities: $(x y) z=x(y z), x x^{-1} x=x$, $\left(x^{-1}\right)^{-1}=x, x x^{-1}=x^{-1} x$.

We introduce notation for the following varieties of completely regular semigroups:

CR -completely regular semigroups,

G - groups,

B -bands,

LRB-left regular bands,

RRB - right regular bands,

LNB-left normal bands,

S -semilattices.

Denote by $U(A)$ the free unary semigroup on the non-empty set $A$. It is the least subsemigroup $T$ in the free semigroup on the alphabet $A \cup\left\{(,)^{-1}\right\}$ with the property that $(u)^{-1} \in T$ provided $u \in T$. For any word $u$ in the alphabet $A \cup\left\{(,)^{-1}\right\}$, we denote by $\hat{u}$ the word obtained from $u$ by deleting all unmatched brackets.

Given a variety $\mathbf{V}$ of completely regular semigroups and an alphabet $A$, the fully invariant congruence on $U(A)$ corresponding to $\mathbf{V}$ is denoted by $\varrho(\mathbf{V}, A)$. It is well known that there is an antiisomorphism between the lattice of varieties of unary semigroups and the lattice of fully invariant congruences on the free unary semigroup on an infinite alphabet. When describing a property of the variety $\mathbf{V}$, we will use $\varrho(\mathbf{V})$ to denote the fully invariant congruence corresponding to $\mathbf{V}$ on an infinite alphabet.

For any word $u \in U(A)$, we introduce the following notations:

$A(u)$ - the content of $u$, that is, the set of all elements in $A$ occurring in $u$,

$0(u)-\hat{w}$ where $w$ is the longest initial segment $v$ of $u$ such that $|A(v)|=|A(u)|-1$,

$h(u)$ - the head of $u$, that is, the element of $A$ occurring first in $u$ from the left.

If $Q$ is any of $A$ and $h$ then define the equivalence relation

$$
\{(u, v) \in U(A) \times U(A): Q(u)=Q(v)\}
$$

and denote it by $\Delta$ and $h$, respectively. Moreover, put

$$
\Delta^{\prime}=\{(u, v) \in U(A) \times U(A):|A(u)|=|A(v)| \text { and }|A(u)-A(v)| \leqq 1\} .
$$

Given a congruence $\varrho$ on $U(A)$, we define the relation $\varrho_{0}$ as follows:

$$
\varrho_{0}=\left\{(0(u), 0(v)): u, v \in A^{+},|A(u)|,|A(v)| \geqq 2 \text { and } u \varrho v\right\} .
$$

Obviously, $\varrho \subseteq \varrho_{0}$.

The next result follows from [11, Theorem 3(4)(5) and Lemmas 5(1) and 6].

Result 2.1. Let $\mathbf{V}$ be a variety of completely regular semigroups. Then just one of the following conditions holds: 
(i) $(\varrho(\mathbf{V}))_{0}=\Delta^{\prime}$,

(ii) $(\varrho(\mathbf{V}))_{0}=h \cap \Delta^{\prime}$,

(iii) $(\varrho(\mathbf{V}))_{0} \subseteq \Delta$, in which case $(\varrho(\mathbf{V}))_{0}$ is fully invariant congruence and $\left((\varrho(\mathbf{V}))_{0}\right)_{0}=$ $(\varrho(\mathbf{V}))_{0}$.

In case (iii), the variety of completely regular semigroups corresponding to the fully invariant congruence $(\varrho(\mathbf{V}))_{0}$ is denoted by $\mathbf{V}_{0}$. Moreover, we note that if $(\varrho(\mathbf{V}))_{0} \subseteq \Delta$ then one can easily see that $(\varrho(\mathbf{V}))_{0} \subseteq h$ is also valid.

Pastijn [8] noticed that the variety $V_{0}$ is closely related to the variety $V_{T_{r}}$ defined in the following way. Let $U$ stand for the free unary semigroup on an infinite alphabet. If $\varrho$ and $\vartheta$ are fully invariant congruences on $U$ with $\varrho(\mathbf{C R}) \subseteq \varrho, \vartheta$ then we put

$$
\varrho T_{r} \vartheta \quad \text { if and only if } \varrho / \varrho(\mathbf{C R}) \vee \mathscr{R}_{U / \varrho(\text { CR })}=\vartheta / \varrho(\mathbf{C R}) \vee \mathscr{R}_{U / \varrho(\mathbf{C R})} .
$$

This relation $T_{r}$ is a complete congruence on the lattice of fully invariant congruences on $U$. Therefore each $T_{r}$-class is an interval. We denote by $\varrho^{T_{r}}$ the greatest element in the interval containing the fully invariant congruence $\varrho$. The variety corresponding to the fully invariant congruence $\varrho(V)^{T_{r}}$ will be denoted by $V_{T_{r}}$.

It is shown in [8, Theorem 2] that $\varrho^{T_{r}} / \varrho(\mathbf{C R})=\left(\varrho / \varrho(\mathbf{C R}) \vee \mathscr{R}_{U / \ell(\mathbf{C R})}\right)^{b}$, the greatest congruence on $U / \varrho(\mathbf{C R})$ contained in $\varrho / \varrho(\mathbf{C R}) \vee \mathscr{R}_{U / \ell(\mathbf{C R})}$. This implies by Result 1.1 that

$$
\varrho(\mathbf{V})^{T_{r}} / \varrho(\mathbf{V})=\mathscr{R}_{U / \varrho(\mathbf{V})}^{b}
$$

Combining Result 2.1 and [8, Lemmas 6 and 7], we infer the following statement.

Result 2.2. For any variety $\mathrm{V}$ of completely regular semigroups, we have

(i) $(\varrho(\mathbf{V}))_{0}=\Delta^{\prime} \Leftrightarrow(\varrho(\mathbf{V}))_{0} \nsubseteq h \Leftrightarrow \mathbf{V}_{T_{r}}=\mathbf{S}$,

(ii) $(\varrho(\mathbf{V}))_{0}=h \cap \Delta^{\prime} \Leftrightarrow(\varrho(\mathbf{V}))_{0} \subseteq h$ and $(\varrho(\mathbf{V}))_{0} \nsubseteq \Delta \Leftrightarrow \mathbf{V}_{T_{r}}=\mathbf{L N B}$,

(iii) $(\varrho(\mathbf{V}))_{0} \subseteq \Delta \Rightarrow \mathbf{V}_{0}=V_{T_{r}}$.

The following lemma finds connection between the members of $\mathbf{V}$ and $\mathbf{V}_{T_{r}}$.

Lemma 2.3. Let $\mathrm{V}$ be a variety of completely regular semigroups. If $S \in \mathrm{V}$ then $S / \mathscr{R}_{S}^{b} \in \mathbf{V}_{T_{r}}$.

Proof. Since $S \in \mathbf{V}$, we have a surjective homomorphism $\phi: U / \varrho(\mathbf{V}) \rightarrow S$ for some free unary semigroup $U$. By $(2.1)$ we see that $(U / \varrho(\mathbf{V})) / \mathscr{R}_{U / \ell(\mathrm{V})}^{b}$ is isomorphic to $U / \varrho(\mathbf{V})^{T_{r}}$ which belongs to $\mathbf{V}_{r_{r}}$. However, it is standard to verify that if $\chi: T \rightarrow S$ is a surjective homomorphism then $\mathscr{R}_{T}^{b} \subseteq \equiv_{\chi\left(\mathscr{A}_{S}^{b}\right)^{\mathrm{t}}}$, and so there exists a unique homomorphism $\chi^{\prime}: T / \mathscr{R}_{T}^{b} \rightarrow S / \mathscr{R}_{S}^{b}$ with $\chi\left(\mathscr{R}_{S}^{b}\right)^{\natural}=\left(\mathscr{R}_{T}^{b}\right)^{\natural} \chi^{\prime}$. Clearly, $\chi^{\prime}$ is also surjective. Applying this for $T=U / \varrho(\mathbf{V})$, we obtain that $S / \mathscr{R}_{\mathrm{S}}^{\mathrm{b}}$ is a homomorphic image of a member in $\mathrm{V}_{T_{r}}$, and so, indeed, $S / \mathscr{R}_{S}^{b} \in \mathbf{V}_{T_{r}}$.

Combining Result 2.2 and Lemma 2.3, we infer the following lemma. 
Lemma 2.4. Let $\mathrm{V}$ be a variety of completely regular semigroups. If $S \in \mathrm{V}$ then

$$
S / \mathscr{R}_{S}^{\mathrm{b}} \in \begin{cases}\mathbf{S}, & \text { if }(\varrho(\mathbf{V}))_{0} \nsubseteq h, \\ \mathbf{L N B}, & \text { if }(\varrho(\mathbf{V}))_{0} \subseteq h \text { and }(\varrho(\mathbf{V}))_{0} \nsubseteq \Delta, \\ \mathbf{V}_{0}, & \text { if }(\varrho(\mathbf{V}))_{0} \subseteq \Delta .\end{cases}
$$

Now we are able to prove the main result in this section.

Theorem 2.5. Let $\mathbf{V}$ be a variety of completely regular semigroups. Let $S$ be a regular semigroup and $\theta$ a group congruence on $S$ such that $\operatorname{ker} \theta \in \mathrm{V}$. Put $\zeta=\mathscr{R}_{S}^{b} \cap \theta$. Then $\theta / \zeta$ is a group congruence on $S / \zeta$ such that $(S / \zeta) /(\theta / \zeta)$ is isomorphic to $S / \theta$, and

$$
\operatorname{ker}(\theta / \zeta) \in \begin{cases}\mathbf{S}, & \text { if }(\varrho(\mathbf{V}))_{0} \nsubseteq h, \\ \mathbf{L N B}, & \text { if }(\varrho(\mathbf{V}))_{0} \subseteq h \text { and }(\varrho(\mathbf{V}))_{0} \nsubseteq \Delta, \\ \mathbf{V}_{0}, & \text { if }(\varrho(\mathbf{V}))_{0} \subseteq \Delta\end{cases}
$$

Proof. For brevity, denote $\operatorname{ker} \theta$ by $K$. Then $\operatorname{ker}(\theta / \zeta)=K /(\zeta \mid K)$. We intend to prove that $\mathscr{R}_{K}^{b} \subseteq \zeta \mid K$. Since $\theta \mid K=K \times K$, it suffices to show that $\mathscr{R}_{K}^{b} \subseteq \mathscr{R}_{S}^{b} \mid K$. Let $k, l \in K$ such that $k \mathscr{R}_{K}^{b} l$. In order to show that $k \mathscr{R}_{S}^{b} l$, we have to verify that $k s \mathscr{R}_{S} l s$ for every $s \in S$. However, $k s \mathscr{R}_{S} k s s^{\prime}$ and $l s \mathscr{R}_{S} l s s^{\prime}$ for any $s^{\prime} \in V(s)$. Since $s s^{\prime} \in E \subseteq K$, we have $k s s^{\prime} \mathscr{R}_{K}^{b} l s s^{\prime}$. This implies $k s s^{\prime} \mathscr{R}_{s} l s s^{\prime}$ because $\mathscr{R}_{K}^{b} \subseteq \mathscr{R}_{K} \subseteq \mathscr{R}_{S}$. Thus we infer that $k s \mathscr{R}_{s} l s$ which completes the proof of the inclusion $\mathscr{R}_{K}^{b} \subseteq \zeta \mid K$.

The inclusion implies that $\operatorname{ker}(\theta / \zeta)$ is a homomorphic image of $K / \mathscr{R}_{K}^{b}$ where $K \in \mathbf{V}$. However, by Lemma 2.4 , we obtain that $K / \mathscr{R}_{K}^{b}$ belongs to $S, \mathbf{L N B}$ and $\mathbf{V}_{0}$ according to $(\varrho(\mathbf{V}))_{0} \nsubseteq h,(\varrho(\mathbf{V}))_{0} \subseteq h$ and $(\varrho(\mathbf{V}))_{0} \nsubseteq \Delta$, and $(\varrho(\mathbf{V}))_{0} \subseteq \Delta$, respectively. This completes the proof.

In Section 4 we will apply this theorem for band varieties. We conclude this section with recalling the recursive solution of the word problem for the free objects in band varieties. First of all, we notice that a band is a completely regular semigroup in which the identity $a^{-1}=a$ holds. So, when working with band varieties, the unary operation -1 and the troubles with unmatched brackets can be eliminated and $U(A)$ can be substituted by $A^{+}$. The notations $A(u), 0(u), h(u), \Delta$ and $h$, correspondingly simplified, will be used in this case. Furthermore, we introduce, for any $u \in A^{+}$,

$$
\begin{aligned}
& \bar{O}(u) \text {-the element } a \in A \text { such that } O(u) a \text { is an initial segment of } u \text {, } \\
& l(u) \text { - the shortest initial segment } v \text { of } u \text { such that }|A(v)|=|A(u)| .
\end{aligned}
$$

Note that $l(u)=0(u) \overline{0}(u)$. Moreover, dually to $0(u), \overline{0}(u)$ and $h(u)$, we define $1(u), \overline{1}(u)$ and $t(u)$ (called the tail of $u$ ), respectively. Analogously to the relations $h$ and $\varrho_{0}$, we introduce also the relations $t$ and $\varrho_{1}$, respectively. 
The following recursive solution of the word problem for the free objects in band varieties can be obtained by [12, Theorem 1.3(1)].

Result 2.6. Let $\mathrm{V}$ be a band variety such that $\mathrm{S} \subseteq \mathrm{V}$ and let $A$ be a non-empty set. Then, for any $u, v \in A^{+}$, we have $u \varrho(V, A) v$ if and only if the following conditions are satisfied:

(i) $A(u)=A(v)$,

(ii) if $(\varrho(\mathbf{V}, A))_{0} \subseteq \Delta$ then $\overline{0}(u)=\overline{0}(v)$ and, in case $|A(u)| \geqq 2,0(u)(\varrho(\mathbf{V}, A))_{0} 0(v)$,

(iii) if $(\varrho(\mathbf{V}, A))_{1} \subseteq \Delta$ then $\overline{1}(u)=\overline{1}(v)$ and, in case $|A(u)| \geqq 2,1(u)(\varrho(\mathbf{V}, A))_{1} 1(v)$,

(iv) if $(\varrho(\mathbf{V}, A))_{0} \subseteq h$ and $(\varrho(\mathbf{V}, A))_{0} \nsubseteq \Delta$ then $h(u)=h(v)$,

(v) if $(\varrho(\mathbf{V}, A))_{1} \subseteq t$ and $(\varrho(\mathbf{V}, A))_{1} \nsubseteq \Delta$ then $t(u)=t(v)$,

In particular, one can easily derive the following description of $\varrho(\operatorname{LNB}, A)$ and $\varrho(\mathbf{L R B}, A)$ from the previous result (cf. also [9]).

Result 2.7. For any non-empty set $A$, we have

$$
\varrho(\mathbf{L N B}, A)=\left\{(u, v): u, v \in A^{+}, A(u)=A(v) \text { and } h(u)=h(v)\right\}
$$

and

$$
\varrho(\mathbf{L R B}, A)=\left\{(u, v): u, v \in A^{+}, A(u)=A(v) \text { and } i(u)=i(v)\right\}
$$

where $i(u)$, the initial part of $u$ is the word obtained from $u$ by retaining only the first occurrence of each letter.

\section{Quasi-F-orthodox semigroups}

In order to generalize $F$-inverse semigroups, Edwards [1] investigated the regular semigroups whose $\sigma$-classes contain greatest elements with respect to the natural partial order. Since these semigroups are orthodox monoids, she called them $F$-orthodox semigroups. Moreover, she also verified that an $F$-orthodox semigroup is necessarily E-unitary.

Given an $F$-orthodox semigroup $S$, we will denote by $t_{g}$ the greatest element in the $\sigma$-class $g$ for every $g \in G$. In particular, $t_{1}$ is the identity in $S$.

If $S$ is an $E$-unitary regular semigroup and $\varepsilon$ is an idempotent pure congruence on $S$ then $\varepsilon \subseteq \sigma$ and $\sigma / \varepsilon$ is the least group congruence on $S / \varepsilon$. Clearly, $\sigma / \varepsilon$ is idempotent pure and hence $S / \varepsilon$ is also $E$-unitary. Furthermore, the mapping $G_{S} \rightarrow G_{S / \varepsilon}, s \sigma \mapsto(s \varepsilon)(\sigma / \varepsilon)$ is an isomorphism. If $g \in G_{s}$ and $g=s \sigma$ for some $s \in S$ then we write $g^{g}$ for $(s \varepsilon)(\sigma / \varepsilon)$.

Now we deal with the idempotent pure homomorphic images of an $F$-orthodox semigroup.

Lemma 3.1. Let $S$ be an F-orthodox semigroup and $\varepsilon$ an idempotent pure congruence on $S$. Then we have $t_{g^{\varepsilon}}=t_{g} \varepsilon$ in $S / \varepsilon$ for every $g \in G_{S}$. Consequently $S / \varepsilon$ is $F$-orthodox. 
Proof. By Result 1.4, we see that $t_{g} \varepsilon$ is, indeed, the greatest element in $g^{\varepsilon}$ for every $g \in G$.

In particular, since $\gamma$ is idempotent pure, we obtain from Lemma 3.1 that the greatest inverse semigroup homomorphic image of an $F$-orthodox semigroup is an $F$-inverse semigroup. By means of this property, we introduce a notion generalizing that of an $F$ orthodox semigroup.

An orthodox semigroup $S$ will be termed quasi-F-orthodox if the greatest inverse semigroup homomorphic image of $S^{1}$ is $F$-inverse. We obviously have the following corollary.

Corollary 3.2. Each F-orthodox semigroup is quasi-F-orthodox.

It is well known that the free inverse monoids are $F$-inverse semigroups. Since the greatest inverse semigroup homomorphic image of a bifree orthodox semigroup is a free inverse semigroup, hence it follows that each bifree orthodox semigroup is quasi- $F$ orthodox. However, we will see at the end of this section that the bifree orthodox monoids are not $F$-orthodox. In fact, quasi- $F$-orthodox semigroups were found to be worth investigating because they possess the following property $(R)$ which, in the special case of the bifree orthodox semigroups, plays a crucial role in the proof of the embedding theorem in [14]:

$(R)$ each non-identity $\sigma$-class $g \in G$ contains an element $r_{g}$ such that $s r_{g} s=s$ for every $s \in g^{-1}$.

Proposition 3.3. For an orthodox semigroup $S$, the following two conditions are equivalent:

(i) $S$ is quasi-F-orthodox.

(ii) $S$ is E-unitary and possesses property $(R)$.

Proof. Assume first that $S$ is quasi- $F$-orthodox. Then $S$ is $E$-unitary. For, $S^{1} / \gamma$ is $F$-inverse and hence $E$-unitary. This implies $S^{1}$ to be $E$-unitary, and so $S$ is also $E$-unitary. Now we show that $S$ has property $(R)$. Again utilizing that $S^{1} / \gamma$ is $F$-inverse, for every $g \in G, g \neq 1$, there exists $r \in S$, such that $r \gamma$ is the greatest element in the $(\sigma / \gamma)$ class $g^{\gamma}$. Then, by Result 1.3 , we clearly have $s r s \gamma s$ for every $s \in g^{-1}$. However, since $s r s \in s S \cap S s$, we obtain that $s r s=s$ is valid. Thus $S$ has property $(R)$.

Conversely, suppose that $S$ is $E$-unitary and possesses property $(R)$. This implies by Result 1.3 that $\left(r_{g-1}, \gamma\right)^{-1}$ is the greatest element in the $(\sigma / \gamma)$-class of $s \gamma$, that is, in $g^{\gamma}$. Taking into account that $S$ is $E$-unitary, the identity in $S^{1} / \gamma$ is the greatest element in the identity $(\sigma / \gamma)$-class. Thus $S^{1} / \gamma$ is shown to be $F$-inverse, and so $S$ is quasi- $F$-orthodox.

Notice that, in this proof, we have verified the following lemma:

Lemma 3.4. Let $S$ be a quasi-F-orthodox semigroup. For every $g \in G$ with $g \neq 1$, the set 


$$
\hat{g}=\left\{r \in g: s r s=s \quad \text { for every } \quad s \in g^{-1}\right\}
$$

constitutes a $\gamma$-class. In fact, $\hat{g}$ is the greatest element in the $(\sigma / \gamma)$-class $g^{\gamma}$.

Let $S$ be a quasi- $F$-orthodox semigroup. By an $F$-cross-section in $S$, we mean a mapping $R: G \backslash\{1\} \rightarrow S$ such that $g R \in \hat{g}$ for every $g \in G, g \neq 1$. An extended F-cross-section in $S$ is defined to be a mapping $R: G \rightarrow S^{1}$ such that $R \mid G \backslash\{1\}$ is an $F$-cross-section in $S$ and $1 R=1$. Clearly, each $F$-cross-section $R$ in $S$ can be uniquely extended to an extended $F$-cross-section which we will denote by $R^{1}$. We will find it convenient to denote the $F$-cross-section $R$ and the extended $F$-cross-section $R$ also by $(g R: g \in G, g \neq 1)$ and $(g R: g \in G)$, respectively.

In particular, if $S$ is $F$-orthodox, then $T_{S}=\left(t_{g}: g \in G\right)$ is the unique extended $F$-crosssection in $S$. The analogue of Lemma 3.1 holds for quasi- $F$-orthodox semigroups and for $F$-cross-section in them.

Lemma 3.5. Let $S$ be a quasi-F-orthodox semigroup and $R=\left(r_{g}: g \in G_{S}, g \neq 1\right)$ an $F$-cross-section in $S$. Let $\varepsilon$ be an idempotent pure congruence on $S$. Then $R^{\varepsilon}=$ $\left(\bar{r}_{g^{r}}: g^{\varepsilon} \in G_{S / \varepsilon}, g^{\varepsilon} \neq 1\right)$ with $\bar{r}_{g^{r}}=r_{g} \varepsilon(g \in G, g \neq 1)$ is an F-cross-section in $S / \varepsilon$. Consequently, $S / \varepsilon$ is quasi-F-orthodox.

Proof. It is straightforward to check that, since $r_{g} \in \hat{g}$ for every $g \in G_{S}, g \neq 1$, we have $r_{g} \varepsilon \in \hat{g}^{\varepsilon}$ for every $g^{\varepsilon} \in G_{S / \varepsilon}, g^{\varepsilon} \neq 1$. Thus $R^{\varepsilon}$ is, indeed, an $F$-cross-section. Since $S / \varepsilon$ is $E$-unitary, Proposition 3.3 implies that $S / \varepsilon$ is quasi- $F$-orthodox.

In connection with the extended $F$-cross-sections, we can add the following remark to Lemma 3.5 .

Remark 3.6. If $S$ is a quasi-F-orthodox semigroup, $R=\left(r_{g}: g \in G_{S}, g \neq 1\right)$ is an $F$-cross-section in $S$ and $\varepsilon$ is an idempotent pure congruence on $S$, then $\left(R^{\varepsilon}\right)^{1}=\left(R^{1}\right)^{\varepsilon^{1}}$ where $\left(R^{1}\right)^{\varepsilon^{1}}=\left(\bar{r}_{g^{r^{1}}}: g^{c^{1}} \in G_{S^{1} / \varepsilon^{1}}\right)$ with $\bar{r}_{g^{r^{1}}}=r_{g} \varepsilon^{1}(g \in G)$.

Now we turn to introducing the notion of a generalized $F$-orthodox semigroup.

Let $S$ be an $\mathscr{R}$-unipotent quasi- $F$-orthodox semigroup and $R=\left(r_{g}: g \in G\right)$ an extended $F$-cross-section in $S$. We say that $R$ is left normal if $s r_{g}=t r_{h}$ holds for any $s, t \in S$ and $g, h \in G$ such that $s \mathscr{R} t$ and $s \sigma \cdot g=t \sigma \cdot h$.

The following lemma justifies this terminology.

Lemma 3.7. Let $S$ be an (R्R-unipotent) quasi-F-orthodox semigroup whose band of idempotents is left normal. Then each extended F-cross-section in $S$ is left normal.

Proof. Let $R=\left(r_{g}: g \in G\right)$ be an extended $F$-cross-section in $S$ and let $s, t \in S$ and $g, h \in G$ such that $s \mathscr{R} t$ and $s \sigma \cdot g=t \sigma \cdot h$. Since $S$ is $\mathscr{R}$-unipotent, the first relation implies that $s s^{\prime}=t t^{\prime}$ for any $s^{\prime} \in V(s)$ and $t^{\prime} \in V(t)$. By applying to $S^{1} / \gamma$ the well-known structure theorem [10, VII.6.9] for $F$-inverse semigroups, we can easily check that $s r_{g} r_{g^{-1}} s^{\prime}$ and 
$t r_{h} r_{h-1} t^{\prime}$ are $\mathscr{D}$-related idempotents in $S$. Thus, since $s r_{g} \mathscr{R} s r_{g} r_{g^{-1}} s^{\prime}=\left(s s^{\prime}\right)\left(s r_{g} r_{g^{-1}} s^{\prime}\right)$ and, similarly, $t r_{h} \mathscr{R}\left(t t^{\prime}\right)\left(t r_{h} r_{h-1} t^{\prime}\right)$, we infer by Result 1.5(i) that $s r_{g} \mathscr{R} t r_{h}$. However, the assumption $s \sigma \cdot g=t \sigma \cdot h$ implies also $s r_{g} \sigma t r_{h}$. Hence we see by Result 1.7 that $s r_{g}=t r_{h}$. The proof is complete.

A similar assertion holds for $\mathscr{R}$-unipotent $F$-orthodox semigroups.

Lemma 3.8. Let $S$ be an $\mathscr{R}$-unipotent $F$-orthodox semigroup. Then the unique extended $F$-cross-section in $S$ is left normal.

Proof. The unique extended $F$-cross-section in $S$ is $\left(t_{g}: g \in G\right)$. Suppose that $a, b \in S$ and $g, h \in G$ such that $a \mathscr{R} b$ and $a \sigma \cdot g=b \sigma \cdot h$. In the same way as in the previous lemma, it suffices to show that $a t_{g} \mathscr{R} b t_{h}$. Since $a \mathscr{R} b$ by assumption, there exists $u \in S$ such that $a=b u$. Therefore we have $a t_{g}=b u t_{g}$ where $\left(u t_{g}\right) \sigma=h$. Hence $u t_{g} \leqq t_{h}$, and so $u t_{g} \leqq_{g} t_{h}$ follows. This implies $a t_{g} \leqq_{\mathfrak{R}} b t_{h}$. Changing the roles of $a$ and $b$, we see that $b t_{h} \leqq_{\mathfrak{R}} a t_{g}$ also holds, completing the proof.

Now we will show that left normality of an extended $F$-cross-section is preserved by forming idempotent pure homomorphic images.

Lemma 3.9. Let $S$ be an $\mathscr{R}$-unipotent quasi-F-orthodox semigroup and $\varepsilon$ an idempotent pure congruence on it. If $R$ is an F-cross-section in $S$ such that $R^{1}$ is left normal then $\left(R^{\varepsilon}\right)^{1}$ is a left normal extended $F$-cross-section in $S / \varepsilon$.

Proof. Put $R=\left(r_{g}: g \in G_{S}, g \neq 1\right)$. Let $\bar{s}, \bar{t} \in S^{1} / \varepsilon^{1}$ with $\bar{s} \mathscr{R} E$. By Result 1.1 , there exist $s \in \bar{s}$ and $t \in \bar{t}$ such that $s \mathscr{R} t$ in $S^{1}$. Let $g, h \in G_{S}$ with $s \sigma \cdot g=t \sigma \cdot h$, or, equivalently, $g^{\varepsilon^{1}}, h^{\varepsilon^{1}} \in G_{S^{1} / \varepsilon^{1}}$ with $\bar{s}\left(\sigma^{1} / \varepsilon^{1}\right) \cdot g^{\varepsilon^{1}}=\tilde{t}\left(\sigma^{1} / \varepsilon^{1}\right) \cdot h^{\varepsilon^{1}}$. Then the equality $s r_{g}=t r_{h}$, which is valid since $R^{1}$ is left normal, implies $\bar{s} \bar{r}_{g^{f^{1}}}=s \varepsilon^{1} \cdot r_{g} \varepsilon^{1}=t \varepsilon^{1} \cdot r_{h} \varepsilon^{1}=\overline{t r}_{h^{6^{1}}}$. Hence, by Remark 3.6, we see that $\left(R^{c}\right)^{1}$ is also left normal which completes the proof.

Dually to the notion of a left normal extended $F$-cross-section in an $\mathscr{R}$-unipotent semigroup, we can introduce that of a right normal extended $F$-cross-section in an $\mathscr{L}$ unipotent semigroup.

Let $S$ be a quasi- $F$-orthodox semigroup. Recall that $\alpha, \beta \subseteq \sigma$ and hence $\alpha$ and $\beta$ are idempotent pure. We will term $S$ a generalized $F$-orthodox semigroup if there exists an $F$ cross-section $R$ in $S$ such that $\left(R^{\alpha}\right)^{1}$ is left normal and $\left(R^{\beta}\right)^{1}$ is right normal.

We can strengthen Corollary 3.2 as follows.

Proposition 3.10. Each F-orthodox semigroup is generalized F-orthodox.

Proof. Let $S$ be an $F$-orthodox semigroup. Corollary 3.2 shows that $S$ is quasi- $F$ orthodox. By Lemma 3.1 and Remark 3.6, $S / \alpha$ is an $\mathscr{R}$-unipotent $F$-orthodox semigroup and $T_{S / \alpha}=T_{S}^{\alpha}$ where $\alpha=\alpha^{1}$. Lemma 3.8 implies that $T_{S / \alpha}$ is left normal, and so $T_{S}^{\alpha}$ is also left normal. Similarly, we can show that $T_{S}^{\beta}$ is right normal which completes the proof. 
Finally, we verify the analogue of Lemmas 3.1 and 3.5 for generalized $F$-orthodox semigroups.

Lemma 3.11. Let $S$ be a generalized $F$-orthodox semigroup and $\varepsilon$ an idempotent pure congruence on $S$. Suppose that $R$ is an F-cross-section in $S$ such that $\left(R^{\alpha}\right)^{1}$ is left normal and $\left(R^{\beta}\right)^{1}$ is right normal. Then $\left(\left(R^{\varepsilon}\right)^{(\alpha \vee \varepsilon) / \varepsilon}\right)^{1}$ is a left normal extended $F$-cross section in $(S / \varepsilon) /((\alpha \vee \varepsilon) / \varepsilon)$ and $\left(\left(R^{\varepsilon}\right)^{(\beta \vee \varepsilon) / \varepsilon}\right)^{1}$ is a right normal extended F-cross-section in $(S / \varepsilon) /$ $((\beta \vee \varepsilon) / \varepsilon)$ where $(\alpha \vee \varepsilon) / \varepsilon$ and $(\beta \vee \varepsilon) / \varepsilon$ are the least $\mathscr{R}$-unipotent and the least $\mathscr{L}$ unipotent congruences, respectively, on $S / \varepsilon$. Consequently, $S / \varepsilon$ is generalized $F$-orthodox.

Proof. It is obvious that $(\alpha \vee \varepsilon) / \varepsilon$ is the least $\mathscr{R}$-unipotent congruence on $S / \varepsilon$. Moreover, $\alpha \vee \varepsilon$ is an idempotent pure $\mathscr{R}$-unipotent congruence on $S$, and $\varphi$ : $S /(\alpha \vee \varepsilon) \rightarrow(S / \alpha) /((\alpha \vee \varepsilon) / \alpha),(S(\alpha \vee \varepsilon)) \varphi=(s \alpha)((\alpha \vee \varepsilon) / \alpha)$ and $\psi: S /(\alpha \vee \varepsilon) \rightarrow(S / \varepsilon) /((\alpha \vee \varepsilon) / \varepsilon)$, $(s(\alpha \vee \varepsilon)) \psi=(s \varepsilon)((\alpha \vee \varepsilon) / \varepsilon)$ are isomorphisms. Since $\left(R^{\alpha}\right)^{1}$ is left normal by assumption, Lemma 3.9 ensures that $\left(\left(R^{\alpha}\right)^{(\alpha \vee \varepsilon) / \alpha}\right)^{1}$ is also left normal. Applying the isomorphism $\varphi^{-1} \psi$, which maps the members of $\left(R^{\alpha}\right)^{(\alpha \vee \varepsilon) / \alpha}$ just into those of $\left(R^{\varepsilon}\right)^{(\alpha \vee \varepsilon) / \varepsilon}$, one can immediately see that $\left(\left(R^{\varepsilon}\right)^{(\alpha \vee \varepsilon) / \varepsilon}\right)^{1}$ must also be left normal. Similarly, we can verify that $\left(\left(R^{\varepsilon}\right)^{(\beta \vee \varepsilon) / \varepsilon}\right)^{1}$ is right normal. Since $S / \varepsilon$ is quasi- $F$-orthodox by Lemma 3.5, we infer that $S / \varepsilon$ is also generalized $F$-orthodox. This completes the proof.

We conclude this section by investigating the properties of certain relatively bifree orthodox semigroups. For completeness, after recalling the main facts concerning evarieties of orthodox semigroups, we present the models of these bifree objects obtained in [5]. We mainly follow the formulation in [14]. For more details, the reader is referred to [5] and [14].

An e-variety (called also bivariety) of regular semigroups is a class of regular semigroups closed under forming direct products, regular subsemigroups and homomorphic images. For example, for any band variety $\mathbf{C}$, the class $\mathbf{O}_{c}$ of all orthodox semigroups whose band of idempotents belongs to $\mathbf{C}$ forms an e-variety. In particular, $\mathbf{O}_{\mathbf{B}}$ is the class of all orthodox semigroups which we will denote simply by $\mathbf{O}$.

Let $A$ be a non-empty set. We "double" it in the following manner. Let $A^{*}$ be a set disjoint from $A$ together with a bijection *: $A \rightarrow A^{*}, a \mapsto a^{*}(a \in A)$, and put $\bar{A}=A \cup A^{*}$. Consider the free semigroup on $\bar{A}$ and denote it by $A^{\oplus}$.

A biidentity in the alphabet $A$ is a pair of words $u \hat{=} v$ with $u, v \in A^{\oplus}$. An orthodox semigroup $S$ satisfies $a$ biidentity $u\left(a_{1}, a_{1}^{*}, \ldots, a_{n}, a_{n}^{*}\right) \triangleq v\left(a_{1}, a_{1}^{*}, \ldots, a_{n}, a_{n}^{*}\right)$ if, for every $s_{1}, \ldots, s_{n} \in S$ and every $s_{1}^{\prime} \in V\left(s_{1}\right), \ldots, s_{n}^{\prime} \in V\left(s_{n}\right)$, we have $u\left(s_{1}, s_{1}^{\prime}, \ldots, s_{n}, s_{n}^{\prime}\right)=$ $v\left(s_{1}, s_{1}^{\prime}, \ldots, s_{n}, s_{n}^{\prime}\right)$ in $S$.

It is proved in [5] that the e-varieties of orthodox semigroups are just the classes of orthodox semigroups defined by biidentities. Moreover, the notion of a biinvariant congruence is introduced and a one-to-one correspondence is found between the evarieties of orthodox semigroups and the biinvariant congruences on an infinite alphabet. Given an e-variety $\mathbf{V}$ of orthodox semigroups and an alphabet $A$, the biinvariant congruence on $A^{\oplus}$ corresponding to $\mathrm{V}$ is defined by

$$
\zeta(\mathbf{V}, A)=\left\{(u, v) \in A^{\oplus} \times A^{\oplus}: \text { the biidentity } u=v \text { is satisfied in } \mathbf{V}\right\}
$$


The factor semigroup $B F \mathrm{~V}(A)=A^{\oplus} / \zeta(\mathrm{V}, A)$ turns out to be the so-called bifree object in $\mathbf{V}$ on the set $A$. Namely, $B F \mathrm{~V}(A)$ together with the mapping $i: \bar{A} \rightarrow B F \mathrm{~V}(A), a \mapsto a \zeta(\mathrm{V}, A)$ have the following property. Given an orthodox semigroup $S \in \mathbf{V}$ and a matched mapping $\vartheta: \bar{A} \rightarrow S$, which means that $a^{*} \vartheta \in V(a \vartheta)(a \in A)$, there exists a unique homomorphism $\phi: B F V(A) \rightarrow S$ such that $\iota \phi=\vartheta$.

A model of $B F \mathbf{O}_{\mathrm{C}}(A)$ yields as follows. Consider the Cayley graph $\mathscr{G}$ of the free group $G=B F \mathbf{G}(A)$. For brevity, denote $w \zeta(\mathbf{G}, A)$ by $\bar{w}$ for every $w \in A^{\oplus}$. The vertices of $\mathscr{G}$ are the elements of $G$ and, for any $g, h \in G$, the set of edges from $g$ to $h$ is

$$
\mathscr{G}(g, h)=\{(g, a) \in G \times A: g \bar{a}=h\} .
$$

We denote the set of all edges in $\mathscr{G}$ by $\operatorname{Arr}(\mathscr{G})$.

For any $(g, a) \in \mathscr{G}(g, h)$, let us add to $\mathscr{G}$ a new edge $\left(h, a^{*}\right)$ from $h$ to $g$ and consider the bijection *: $(g, a) \mapsto\left(h, a^{*}\right)$. So we "double" the graph $\mathscr{G}$. The graph obtained in such a way is denoted by $\overline{\mathscr{G}}$. Consider the free semigroupoid (category without identities) on $\overline{\mathscr{G}}$ and denote it by $\mathscr{G}^{\oplus}$.

The graph $\mathscr{G}$ is a connected tree. Thus, for any two different vertices $g, h \in G$, there exists a unique $(g, h)$-path of minimal length in $\bar{G}$ which we denote by $r(g, h)$. Moreover, define $r(g, g)$ to be the empty path at $g$ for every $g \in G$. Obviously, each $(g, h)$-path contains $r(g, h)$ as a subpath.

Let us interpret $(\operatorname{Arr}(\mathscr{G}))^{\oplus}$ in such a way that $\overline{\operatorname{Arr}(\mathscr{G})}=\operatorname{Arr}(\overline{\mathscr{G}})$ and the bijection of $\operatorname{Arr}(\mathscr{G})$ onto $(\operatorname{Arr}(\mathscr{G}))^{*}$ is the mapping defined in the previous paragraph. Then the paths in $\mathscr{G}^{\oplus}$ can be considered as words in $(\operatorname{Arr}(\mathscr{G}))^{\oplus}$, and the concatenation of non-empty paths in $\mathscr{G}^{\oplus}$ coincides with the concatenation of the respective words in $(\operatorname{Arr}(\mathscr{G}))^{\oplus}$.

A left action of $G$ on $\overline{\mathscr{G}}$ can be defined by putting $h(g, a)=(h g, a)$ for any $g, h \in G$ and $a \in \bar{A}$. This action can be extended to a left action of $G$ on the semigroup $(\operatorname{Arr}(\mathscr{G}))^{\oplus}$ in the usual way which, in particular, determines a left action of $G$ on $\mathscr{G}^{\oplus}$.

Let $\mathbf{C}$ be a band variety. Denote by $\varepsilon_{\mathbf{C}}$ the restriction of $\zeta(\mathbf{C}, \operatorname{Arr}(\mathscr{G}))$ to $\mathscr{G}^{\oplus}$. More precisely, two paths in $\mathscr{G}^{\oplus}$ are defined to be $\varepsilon_{\mathrm{C}}$-related if they are coterminal and they are $\zeta(\mathbf{C}, \operatorname{Arr}(\mathscr{G}))$-related as words. It is easy to see that $\varepsilon_{\mathbf{C}}$ is a congruence on $\mathscr{G}^{\oplus}$ which is compatible with the action of $G$. Consider the subset

$$
S(\mathscr{G}, \mathbf{C})=\left\{p \varepsilon_{\mathbf{C}}: p \in \mathscr{G}^{\oplus}(1,-)\right\}
$$

in the quotient semigroupoid $\mathscr{G}^{\oplus} / \varepsilon_{\mathrm{C}}$. Define a multiplication in the following way:

$$
p \varepsilon_{\mathrm{C}} * q \varepsilon_{\mathrm{C}}=p \varepsilon_{\mathbf{C}} \cdot(\omega(p) q) \varepsilon_{\mathrm{C}} \quad\left(p, q \in \mathscr{G}^{\oplus}(1,-)\right) .
$$

Then $S(\mathscr{G}, \mathrm{C})$ with $l: \bar{A} \rightarrow S(\mathscr{G}, \mathrm{C}), a \mapsto(1, a) \varepsilon_{\mathrm{C}}$ is a bifree object in $\mathbf{O}_{\mathrm{c}}$ on the set $A$. Moreover, $p \varepsilon_{\mathrm{C}}$ and $q \varepsilon_{\mathrm{C}}\left(p, q \in \mathscr{G}^{\oplus}(1,-)\right)$ are congruent modulo the least group congruence on $S(\mathscr{G}, \mathbf{C})$ if and only if $\omega(p)=\omega(q)$.

Proposition 3.12. For any band variety $\mathrm{C}$ and for any non-empty set $A$, (j) the monoid $B F^{1} \mathrm{O}_{c}(A)$ is $F$-orthodox if and only if $\mathrm{C} \subseteq \mathrm{S}$, and 
(ii) the semigroup $B F \mathbf{O}_{\mathrm{C}}(A)$ is generalized $F$-orthodox.

Proof. The "if" part of (i) is well known. Now let $\mathrm{C}$ be a band variety and $A$ a non-empty set. Observe that $R_{\mathrm{C}}=\left(r(1, g) \varepsilon_{\mathrm{C}}: g \in G, g \neq 1\right)$ is an $F$-cross-section in $B F \mathbf{O}_{\mathrm{C}}(A)$. For, one can immediately see that $p \cdot r\left(g^{-1}, 1\right) \cdot p \varepsilon_{\mathrm{C}} p$, that is, $p \varepsilon_{\mathrm{C}} * r(1, g) \varepsilon_{\mathrm{C}} * p \varepsilon_{\mathrm{C}}=p \varepsilon_{\mathrm{C}}$ is valid for every $g \in G, g \neq 1$ and for every $p \in \mathscr{G}^{\oplus}\left(1, g^{-1}\right)$. Since $B F \mathbf{O}_{\mathrm{C}}(A)$ is $E$-unitary, hence it follows by Proposition 3.3 that $B F \mathbf{O}_{\mathrm{C}}(A)$ is quasi- $F$-orthodox.

In order to prove the "only if" part of (i), assume that $B F^{1} \mathbf{O}_{\mathrm{C}}(A)$ is $F$-orthodox. Then $R_{\mathrm{C}}$ is the unique $F$-cross-section in it. Let $a \in A$ and consider the path $p=\left(1, a^{*}\right)\left(\overline{a^{*}}, a\right)(1, a)$ in $\mathscr{G}^{\oplus}$. Clearly, $(1, a)=r(1, \bar{a})$ and $p \varepsilon_{\mathbf{C}},(1, a) \varepsilon_{\mathrm{C}}$ are in the same congruence class modulo the least group congruence on $S(\mathscr{G}, \mathrm{C})$. Thus the inequality $p \varepsilon_{\mathbf{C}} \leqq(1, a) \varepsilon_{\mathbf{C}}$ must hold. This implies $p \varepsilon_{\mathbf{C}} \leqq g(1, a) \varepsilon_{\mathbf{C}}$ whence $(1, a)(1, a)^{*} p \varepsilon_{\mathbf{C}} p$ follows. Here the edge $(1, a)$ is different from both $\left(1, a^{*}\right)$ and $\left(\overline{a^{*}}, a\right)=\left(1, a^{*}\right)^{*}$, so we see that the biidentity $x x^{*} y y^{*} x=y y^{*} x$ holds in $\mathbf{C}$. Thus we obtain that $\mathbf{C} \subseteq \mathbf{R R B}$. A dual argument shows that $\mathbf{C} \subseteq \mathbf{L R B}$ also holds whence we infer that $\mathbf{C} \subseteq \mathbf{S}$.

(ii) Since $B F \mathbf{O}_{\mathbf{C}}(A)$ is an idempotent pure homomorphic image of $B F \mathbf{O}(A)$, it suffices to show by Lemma 3.11 that $B F \mathbf{O}(A)$ is generalized $F$-orthodox. For brevity, denote $R_{\mathbf{B}}$ and $\varepsilon_{\mathrm{B}}$ by $R$ and $\varepsilon$, respectively. By the observation at the beginning of the proof, all we have to prove is that $\left(R^{\alpha}\right)^{1}$ is left normal and $\left(R^{\beta}\right)^{1}$ is right normal. We will verify the former property, the latter one follows dually. First we determine the least $\mathscr{R}$-unipotent congruence $\alpha$ on $B F \mathbf{O}(A)$. By Result 1.6, we see that $\mathbf{O}_{\mathbf{L R B}}$ is just the class of all $\mathscr{R}$-unipotent semigroups. Thus, by the definition of a bifree object, the mapping $\bar{A} \rightarrow B F \mathbf{O}(A) / \alpha, a \mapsto(a \zeta(\mathbf{O}, A)) \alpha(a \in \bar{A})$ can be uniquely extended to a homomorphism of $B F \mathbf{O}_{\mathrm{LRB}}(A)$ to $B F \mathbf{O}(A) / \alpha$. This implies that $\varepsilon_{\mathrm{LRB}} / \varepsilon \subseteq \alpha$. However, the former relation is an $\mathscr{R}$-unipotent congruence on $B F O(A)$ whence it follows that it must be equal to $\alpha$.

Now we verify that $\left(R^{\alpha}\right)$ is left normal. We should prove that if $p \in \mathscr{G}^{\oplus}(1, i)$, $q \in \mathscr{G}^{\oplus}(1, j)(i, j \in G)$, respectively, with

$$
p p^{*} \varepsilon_{\mathbf{L R B}} q q^{*}
$$

and $g, h \in G$ with $i g=j h=k$, say, then we have $p \varepsilon_{\mathrm{LRB}} * r(1, g) \varepsilon_{\mathbf{L R B}}=q \varepsilon_{\mathbf{L R B}} * r(1, h) \varepsilon_{\mathbf{L R B}}$ or, equivalently,

$$
p \cdot r(i, k) \varepsilon_{\mathrm{LRB}} q \cdot r(j, k)
$$

If $i=j$ then we have $p \varepsilon_{\mathbf{L R B}} q$ and this relation clearly holds. Now suppose that $i \neq j$. Let $s$ be the longest common final segment of $r(i, k)$ and $r(j, k), s$ may be also empty. Suppose that $s \in \mathscr{G}^{\oplus}(l, k)$. Then we have

$$
r(i, k)=e_{1} e_{2} \ldots e_{m} s \quad \text { and } \quad r(j, k)=f_{1} f_{2} \ldots f_{n} s
$$

where $e_{u}, f_{v} \in \operatorname{Arr}(\overline{\mathscr{G}}) \quad(1 \leqq u \leqq m, 1 \leqq v \leqq n)$ with $e_{m} \neq f_{n}$. Clearly, $e_{1} e_{2} \ldots e_{m}=r(i, l)$ and $f_{1} f_{2} \ldots f_{n}=r(j, l)$. Thus the latter inequality ensures that $e_{1} e_{2} \ldots e_{m} f_{n}^{*} \ldots f_{1}^{*}=r(i, j)$. By (3.1), the paths $p$ and $q$ span the same subgraph $\mathscr{S}$ in $\mathscr{G}$. Hence $i, j$ are vertices in $\mathscr{S}$ and 
$\mathscr{S}$ is connected. So $r(i, j)$ and, consequently $e_{1} e_{2} \ldots e_{m}$ and $f_{1} f_{2} \ldots f_{n}$ are paths in $\mathscr{S}$. It is easy to see by Result 2.7 that (3.1) implies $p e_{1} e_{2} \ldots e_{m} \varepsilon_{\mathrm{LRB}} q f_{1} f_{2},,, f_{n}$. Since $\varepsilon_{\mathrm{LRB}}$ is a congruence, multiplying on the right with $s$, we obtain the relation (3.2) which was to be proved.

Finally, we summarize the main results of this section in a theorem. Denote by $\mathbf{U}, \mathbf{F}$, $Q F$ and $G F$ the classes of all $E$-unitary regular, $F$-orthodox, quasi- $F$-orthodox and generalized $F$-orthodox semigroups, respectively.

Theorem 3.13. (i) We have $\mathbf{F} \subset \mathbf{G F} \subseteq \mathbf{Q F} \subset \mathbf{U}$.

(ii) All classes F, GF, QF and $\mathbf{U}$ are closed under forming idempotent pure homomorphic images.

(iii) The bifree orthodox semigroup on any non-empty set belongs to GF.

Proof. (iii) follows from Proposition 3.12(ii).

(i) The inclusion $\mathbf{G F} \subseteq \mathbf{Q F}$ follows by definition. The inclusions $\mathbf{F} \subseteq \mathbf{G F}$ and $\mathbf{Q F} \subseteq \mathbf{U}$ are proved in Propositions 3.10 and 3.3, respectively. By (iii) and since $B F O(A)$ has no identity, we see that $B F \mathbf{O}(A) \in \mathbf{G F} \backslash \mathbf{F}$ for every non-empty set $A$. Thus $\mathbf{F} \subset \mathbf{G F}$. The strict inclusion $\mathbf{Q F} \subset \mathbf{U}$ follows from the fact that there exist $E$-unitary inverse monoids that are not $F$-inverse.

(ii) The statement for $\mathbf{U}$ is well known. For $\mathbf{F}, \mathbf{G F}$ and $\mathbf{Q F}$ it is implied by Lemmas $3.1,3.11$ and 3.5 , respectively. The proof is complete.

\section{The embedding theorems}

This section is devoted to proving the main results of the paper.

Theorem 4.1. Each quasi-F-orthodox semigroup is embeddable.

Theorem 4.2. Each generalized F-orthodox semigroup is strictly embeddable.

Observe that each orthodox semigroup whose band of idempotents is rectangular is a rectangular group, that is, a direct product of a rectangular band and a group. Therefore we can restrict ourselves to proving Theorem 4.1 [4.2] for quasi- $F$-orthodox [generalized $F$-orthodox] semigroups whose bands of idempotents are not rectangular.

Let $S$ be a quasi- $F$-orthodox semigroup such that $E$ is not rectangular and let $V$ be a band variety such that $E \in \mathbf{V}$. Then, obviously, we have $\mathbf{S} \subseteq \mathbf{V}$. Recall that $E=\operatorname{ker} \sigma$. Let us construct the semigroupoid $C=C_{S}$. Put $A=\operatorname{Arr}(C)$. Define the label of a non-empty path $p=a_{1} a_{2} \ldots a_{m}\left(a_{1} \in \operatorname{Arr}(C), i=1,2, \ldots, m\right)$ in $C^{+}$by $\operatorname{lab}(p)=s_{1} s_{2},,, s_{m}$ provided $a_{i}=\left(g_{i}, s_{i}\right)(i=1,2, \ldots, m)$. Obviously, we have $\operatorname{lab}(p q)=\operatorname{lab}(p) \operatorname{lab}(q)$ for any consecutive paths $p, q$ in $C$.

Let us choose and fix an $F$-cross-section $R=\left(r_{g}: g \in G, g \neq 1\right)$ in $S$. By means of $R^{1}$, we define arrows in $C^{1}$ connecting the objects: for every $g, h \in G$, we put 


$$
z_{g, h}=\left(g, r_{g-1}\right)
$$

Clearly, $z_{g, h} \in C^{1}(g, h)$. Recall that $z_{g, g}(g \in G)$ is an empty arrow at $g$.

In order to prove that $S$ is embeddable into a semidirect product of a band in $\mathrm{V}$ by a group, we should prove by Result 1.11 that $s \sigma t$ and $(1, s) \tau_{\mathrm{v}}(1, t)$ imply $s=t$ for every $s, t \in S$. The second relation holds by Result 1.9 if and only if there exists a sequence $(1, s)=w_{0}, w_{1}, \ldots, w_{n}=(1, t)$ of words in $A^{+}$such that $w_{i+1}$ is obtained from $w_{i}$ $(i=0,1, \ldots, n-1)$ by one of the rules (S1), (S2) and (S2').

Proof of Theorem 4.1 If we want to prove that $S$ is embeddable, we can choose $V$ to be $\mathbf{B}$, and so, by Remark 1.10 , we can assume that $w_{i+1}$ is obtained from $w_{i}$ $(i=0,1, \ldots, n-1)$ by one of the rules [S1], [S1'], $(\mathrm{S} 2),\left(\mathrm{S} 2^{\prime}\right)$.

Let us connect any word $w$ in $A^{+}$by means of the $z_{g, h}$ 's in the following way: if $w=a_{1} a_{2} \ldots a_{m}$ where $a_{j} \in C\left(g_{j}, h_{j}\right)(j=1,2, \ldots, m)$ then define

$$
\tilde{w}=a_{1} z_{h_{1}, g_{2}} a_{2} z_{h_{2}, g_{3}} a_{3} \ldots a_{m-1} z_{h_{m-1}, g_{m}} a_{m} .
$$

Notice that $\tilde{w} \in C^{+}\left(g_{1}, h_{m}\right)$.

Lemma 4.3. If $w, w^{\prime} \in A^{+}$such that $w^{\prime}$ is obtained from $w$ by one of the rules [S1] and $\left[\mathrm{S}^{\prime}\right]$ then $\alpha(\tilde{w})=\alpha\left(\tilde{w^{\prime}}\right), \omega(\tilde{w})=\omega\left(\widetilde{w^{\prime}}\right)$ and $\operatorname{lab}(\tilde{w})=\operatorname{lab}\left(\widetilde{w^{\prime}}\right)$.

Proof. Without loss of generality we can suppose that $w^{\prime}$ is obtained from $w$ by rule [S1]. The equalities concerning the starting and ending points of the paths are clear. Moreover, we obviously have

$$
\tilde{w}=\tilde{u} z_{g, h} \tilde{x} z_{i, h} \tilde{x} z_{i, j} \tilde{v} \quad \text { and } \quad \widetilde{w^{\prime}}=\tilde{u} z_{g, h} \tilde{x} z_{i, j} \tilde{v}
$$

where $\omega(\tilde{u})=g, \alpha(\tilde{x})=h, \omega(\tilde{x})=i$ and $\alpha(\tilde{v})=j$. In order to check that $\operatorname{lab}(\tilde{w})=\operatorname{lab}\left(\tilde{w}^{\prime}\right)$, it suffices to show that

$$
\operatorname{lab}(\tilde{x})=\operatorname{lab}\left(\tilde{x} z_{i, h} \tilde{x}\right) .
$$

Put $s=\operatorname{lab}(\tilde{x})$. Since $\tilde{x}$ is an $(h, i)$-path, we have $s \sigma=h^{-1} i$. If $h=i$ then $s$ is necessarily an idempotent, and $z_{i, h}$ is an empty arrow whence (4.1) follows. In the opposite case, $\operatorname{lab}\left(z_{i, h}\right)=r_{i-1 h}$. Taking into consideration the definition of $r_{i-1}$, we see that $s r_{i-i_{h}} s=s$ which implies (4.1). This completes the proof.

Lemma 4.4. If $\underset{w}{w} w^{\prime} \in A^{+}$such that $w^{\prime}$ is obtained from $w$ by one of the rules (S2) and (S2') then $\alpha(\tilde{w})=\alpha\left(\widetilde{w^{\prime}}\right), \omega(\tilde{w})=\omega\left(\widetilde{w^{\prime}}\right)$ and $\operatorname{lab}(\tilde{w})=\operatorname{lab}\left(\widetilde{w^{\prime}}\right)$.

Proof. In the same way as above, we can assume that $w^{\prime}$ is obtained from $w$ by rule (S2), and we can restrict ourselves to proving the last equality, the others being obvious. Clearly, we have 


$$
\tilde{w}=\tilde{u} z_{g, i} a z_{j, j} b z_{k, h} \tilde{v} \quad \text { and } \quad \tilde{w^{\prime}}=\tilde{u} z_{g, i} c z_{k, h} \tilde{v}
$$

where $\omega(\tilde{u})=g, a \in C(i, j), b \in C(j, k), c \in C(i, k)$ and $\alpha(\tilde{v})=h$. By definition, $z_{j, j}$ is an empty arrow. Moreover, since $a \circ b=c$, we obtain that $\operatorname{lab}(a b)=\operatorname{lab}(c)$. Hence it follows that $\operatorname{lab}(\tilde{w})=\operatorname{lab}\left(\widetilde{w^{\prime}}\right)$ which completes the proof.

Returning to the proof of Theorem 4.1, Lemmas 4.3 and 4.4 imply that $(1, s)=$ $\widetilde{w_{0}}, \widetilde{w_{1}}, \ldots, \widetilde{w_{n}}=(1, t)$ are $(1, g)$-paths with $g=s \sigma=t \sigma$ and $s=\operatorname{lab}((1, s))=\operatorname{lab}\left(\widetilde{w_{0}}\right)=$ $\operatorname{lab}\left(\widetilde{w_{1}}\right)=\cdots=\operatorname{lab}\left(\widetilde{w_{n}}\right)=\operatorname{lab}((1, t))=t$. Thus the proof of Theorem 4.1 is complete.

The main idea in the proof of Theorem 4.2 is similar. However, we should handle all band varieties. Therefore we will make an induction by applying the recursive solution of the word problem for band varieties formulated in Result 2.6. Since, in the case of certain band varieties, the first or the last letter in a word is not preserved when applying rule (S1), we should modify the definition of the path assigned to a word.

Let $w \in A^{+}$. Suppose that $w=a_{1} a_{2} \ldots a_{m}$ where $a_{i} \in C\left(g_{i}, h_{i}\right)(i=1,2, \ldots, m)$. We assign a subgraph $[w]$ in $C$ to $w$ as follows.

$$
\begin{aligned}
& \operatorname{Obj}([w])=\left\{g_{i}, h_{i}: i=1,2, \ldots, m\right\}, \\
& {[w](j, k)= \begin{cases}C(j, k) \cap A(w) & \text { if } j=k, \\
(C(j, k) \cap A(w)) \cup\left\{z_{j, k}\right\} & \text { if } j \neq k,\end{cases} } \\
& ((j, k) \in \operatorname{Obj}([w])) .
\end{aligned}
$$

Notice that if $A(u)=A(v)$ for some $u, v \in A^{+}$then $[u]=[v]$. Furthermore, we define the notion of a $V$-starting point and a $\mathbf{V}$-ending point of a word $w$. We say that $g \in \operatorname{Obj}(C)$ is a V-starting point of $w$ if $g \in \operatorname{Obj}([w])$ and, in case $\varrho(V) \subseteq h$, also $g=\alpha(h(w))$. Dually, we define a V-ending point of $w$. Put

$$
\begin{gathered}
T_{\mathrm{v}}(w)=\{(g, h): g \text { is a } \mathbf{V} \text {-starting point of } w \text { and } \\
h \text { is a } \mathbf{V} \text {-ending point of } w\} .
\end{gathered}
$$

Clearly, if $u \varrho(V, A) v$ then $T_{\mathbf{V}}(u)=T_{\mathrm{v}}(v)$. For any $(g, h) \in \mathrm{Obj}([w])$, we assign a path in $C$ to $w$ in the following way:

$$
\tilde{w}^{g, h}=z_{g, g_{1}} a_{1} z_{h_{1}, g_{2}} a_{2} z_{h_{2, g}, g_{3}} a_{3} \ldots a_{m-1} z_{h_{m-1}, g_{m}} a_{m} z_{h_{m}, h} .
$$

Notice that $\tilde{w}^{g, h}$ is a $(g, h)$-path in $[w]$.

Now we find connection between $C, A$, lab, ${ }^{-g, h}$ corresponding to $S$ and $C^{\varepsilon}, A^{\varepsilon}$, lab $^{\varepsilon}, \sim^{\varepsilon} g^{\varepsilon}, h^{\varepsilon}$ corresponding to an idempotent pure homomorphic image $S / \varepsilon$ of $S$.

Consider an idempotent pure congruence $\varepsilon$ on $S$. Construct $C^{\varepsilon}, A^{\varepsilon}$ and $\operatorname{lab}^{\varepsilon}$ by means of $S / \varepsilon$ and $\sigma / \varepsilon$. Notice that the morphism $\varphi: C^{+} \rightarrow\left(C^{\varepsilon}\right)^{+}$extending the graph function 


$$
\begin{aligned}
& \operatorname{Obj}(C) \rightarrow \operatorname{Obj}\left(C^{\varepsilon}\right), g \mapsto g^{\varepsilon}, \\
& C(g, h) \rightarrow C^{\varepsilon}\left(g^{\varepsilon}, h^{\varepsilon}\right),(g, s) \mapsto\left(g^{\varepsilon}, s \varepsilon\right) \quad((g, s) \in C(g, h))
\end{aligned}
$$

is a quotient morphism. Moreover, it is clear by definition that $\operatorname{lab}^{\varepsilon}(p \varphi)=(\operatorname{lab}(p)) \varepsilon$ for every $p \in C^{+}$.

Furthermore, defining $z_{g^{r}, h^{\varepsilon}}^{\varepsilon}$ by means of $\left(R^{\varepsilon}\right)^{1}$ and $\tilde{w}^{r^{r} g^{c}, h^{c}}$ by means of the $z_{g^{r}, h^{c}}^{\varepsilon}$ s (as before for $S$ ) for every $w \in\left(A^{c}\right)^{+}$, we see that $\widetilde{w}_{\bar{\varphi}}^{c^{c}, h^{c}}=\tilde{w}^{g, h} \bar{\varphi}$ for every $w \in A^{+}$and $g, h \in \operatorname{Obj}(C)$. Here by $\bar{\varphi}$ we mean the homomorphism $A^{+} \rightarrow\left(A^{c}\right)^{+}$extending the mapping $A \rightarrow A^{\mathrm{c}}, a \mapsto a \varphi$. These observations allow us to apply a statement concerning $C, A$, lab, $\tilde{w}^{g, h}$ for an idempotent pure homomorphic image of $S$.

In particular, the local semigroups in the semigroupoid $C^{\gamma}$ are semilattices. Thus Simon's Theorem ([2]) implies that $\operatorname{lab}^{\gamma}(p)=\operatorname{lab}^{\gamma}(q)$ for any coterminal paths, $p, q$ in $C^{\gamma}$ with $A(p)=A(q)$. By the previous argument, this ensures the following property in $C$.

Lemma 4.5. For any coterminal paths $p, q$ in $C^{+}$with $A(p)=A(q)$, we have $\operatorname{lab}(p) \gamma \operatorname{lab}(q)$.

This property helps us to prove the following statement which will play a crucial role in the sequel.

Lemma 4.6. If $u \in A^{+}, g, h \in \mathrm{Obj}([u])$ and $p$ and $q$ are $(g, h)$-paths in $[u]$ such that $A(u) \subseteq A(p), A(q)$ then $\operatorname{lab}(p) \gamma \operatorname{lab}(q)$. In particular, if $u, v \in A^{+}$with $A(u)=A(v)$ and $g, h \in \operatorname{Obj}([u])$ then $\operatorname{lab}\left(\tilde{u}^{g, h}\right) \gamma \operatorname{lab}\left(\tilde{v}^{g, h}\right)$.

Proof. The second statement immediately follows from the first one because $A(u)=A(v)$ implies $[u]=[v]$. As far as the first statement is concerned, by Lemma 4.5 , it suffices to show that if $r$ is a $(g, h)$-path in $[u]$ with $A(u) \subseteq A(r)$ then there exists a $(g, h)$ path $s$ such that $s$ spans $[u]$ and $\operatorname{lab}(r)=\operatorname{lab}(s)$. Since $\operatorname{Arr}([u]) \backslash A(u)$ contains only edges $z_{i, j}(i, j \in \operatorname{Obj}([u]), i \neq j)$, we can get such an $s$ step by step by applying the following observation. If $r$ is a $(g, h)$-path in $[u]$ such that $A(u) \subseteq A(r)$ then, for every $i, j \in \operatorname{Obj}([u])$ with $i \neq j, r$ is of the form $r_{1} r_{2} r_{3}$ where $r_{2}$ is either an $(i, j)$-path or a $(j, i)$-path. Without loss of generality, we can assume that $r_{2}$ is an $(i, j)$-path. Then $\bar{r}=r_{1} r_{2} z_{j, i} z_{i, j} z_{j, i} r_{2} r_{3}$ is a $(g, h)$-path such that $\operatorname{lab}(\vec{r})=\operatorname{lab}(r)$ and $A(r) \cup\left\{z_{i, j}, z_{j, i}\right\} \subseteq A(\vec{r})$. The proof is complete.

By making use of this lemma, we can easily prove the following statement.

Lemma 4.7. If $w \in A^{+}, g, h, i \in \operatorname{Obj}([w])$ then $\operatorname{lab}\left(\tilde{w}^{g . h}\right) \mathscr{R} \operatorname{lab}\left(\widetilde{l}^{g}(w)^{g . i}\right)$.

Proof. We clearly have $[w]=[l(w)]$. Put $w=l(w) w_{1} \quad\left(A\left(w_{1}\right) \subseteq A(w)=A(l(w))\right), j=$ $\omega(t(l(w)))$ and $k=\alpha\left(h\left(w_{1}\right)\right)$. Then we have 


$$
\begin{aligned}
\operatorname{lab}\left(\tilde{w}^{g \cdot h}\right) \mathscr{R} \operatorname{lab}\left(\tilde{w}^{g \cdot h} z_{h, g}\right) & =\operatorname{lab}\left(\widetilde{l(w)}^{g, j}{ }_{z_{j, k}}{\widetilde{w_{1}}}^{k, h} z_{h, g}\right) \\
& =\operatorname{lab}\left(\widetilde{l(w)}^{g, j}{ }_{z_{j, g}} \operatorname{lab}\left(\widetilde{l(w)^{g, j}} z_{j, k}{\widetilde{w_{1}}}^{k, h} z_{h, g}\right)\right. \\
& =\operatorname{lab}\left(\widetilde{l(w)}^{g, j}{ }_{z_{j, g}}\right) \operatorname{lab}\left(\tilde{w}^{g \cdot h} z_{h, g}\right)
\end{aligned}
$$

and, similarly

$$
\begin{aligned}
& \operatorname{lab}\left(\widetilde{l(w)^{g . i}}\right) \mathscr{R} \operatorname{lab}\left(\widetilde{l(w)}{ }^{g, i} z_{i, g}\right)=\operatorname{lab}\left(\widetilde{l(w)^{g . j}} z_{j, i} z_{i, g}\right) \\
& =\operatorname{lab}\left(\widetilde{l(w)^{g, j}} z_{j, g}\right) \operatorname{lab}\left(\tilde{l(w)}{ }^{g, j} z_{j, i} z_{i, g}\right)
\end{aligned}
$$

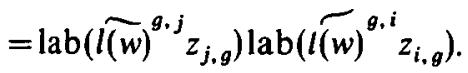

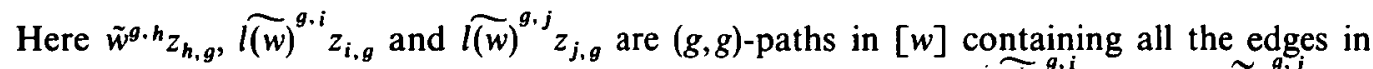
$A(w)$. Therefore Lemma 4.6 ensures that $\operatorname{lab}\left(\tilde{w}^{g \cdot h} z_{h, g}\right) \gamma \operatorname{lab}\left(\widetilde{l(w)}{ }^{g, i} z_{i, g}\right) \gamma \operatorname{lab}\left(l(\widetilde{w})^{g, j} z_{j, g}\right)$. Moreover, these elements are idempotent, so we infer from the previous relations that $\operatorname{lab}\left(\tilde{w}^{g, h}\right) \mathscr{R} \operatorname{lab}\left(\tilde{l}(w)^{g, i}\right)$. The proof is complete.

Now we verify the analogue of Lemma 4.4 .

Lemma 4.8. Let $w, w^{\prime} \in A^{+}$such that $w^{\prime}$ is obtained from $w$ by one of the rules (S2) and $\left(\mathrm{S} 2^{\prime}\right)$. Then $\operatorname{lab}\left(\tilde{w}^{g, h}\right)=\operatorname{lab}\left({\widetilde{w^{\prime}}}^{g \cdot h}\right)$ for every $g, h \in \operatorname{Obj}([w])$.

Proof. It suffices to consider the case when $w^{\prime}$ is obtained from $w$ by rule (S2). Then $w=u a b v, w^{\prime}=u c w$ where $u, v \in\left(A^{+}\right)^{1}$ and $a, b, c \in A$ with $a \circ b=c$. So

$$
\tilde{w}^{g, h}=\tilde{u}^{g, i} a z_{j, j} b \tilde{v}^{k, h} \quad \text { and } \quad{\widetilde{w^{\prime}}}^{g, h}=\tilde{u}^{g, i} c \tilde{v}^{k, h}
$$

provided $a \in C(i, j), b \in C(j, k)$ and $c \in C(i, k)$. Since, by definition, $z_{j, j}$ is an empty arrow, the assumption $a \circ b=c$ immediately implies the required equality.

Before turning to the proof of the analogous result with (S1) we treat the special case $\mathbf{V}=\mathbf{L N B}$ separately.

Lemma 4.9. If $\operatorname{ker} \sigma \in \mathbf{L N B}$ and $w, w^{\prime} \in A^{+}$with $w \varrho(\mathbf{L N B}, A) w^{\prime}$ then $\operatorname{lab}\left(\tilde{w}^{g . h}\right)=$ $\operatorname{lab}\left({\widetilde{w^{\prime}}}^{g, h}\right)$ for every $g, h \in \operatorname{Obj}([w])$.

Proof. Since $w \varrho(\mathrm{LNB}, A) w^{\prime}$ we have $A(w)=A\left(w^{\prime}\right)$ and $h(w)=h\left(w^{\prime}\right)$ by Result 2.7. For brevity, denote $h(w)$ by $a$ and assume that $a \in C(i, j)$. Then $w=a u, w^{\prime}=a v$ and $\tilde{w}^{g, h}=$ $z_{g, i} a \tilde{u}^{j, h}, \widetilde{w}^{\prime g \cdot h}=z_{g, i} a \tilde{v}^{j, h}$. We see that

$$
\operatorname{lab}\left(a \tilde{u}^{j, h}\right) \mathscr{R} \operatorname{lab}\left(a \tilde{u}^{j, h} z_{h, i}\right)=\operatorname{lab}\left(a z_{j, i}\right) \operatorname{lab}\left(a \tilde{u}^{j, h} z_{h, i}\right)
$$

where $e=\operatorname{lab}\left(a z_{j, i}\right)$ and $f=\operatorname{lab}\left(a \tilde{u}^{j, h} z_{h, i}\right)=\operatorname{lab}\left(\tilde{w}^{i, h} z_{h, i}\right)$ are idempotents in $S$. Thus we obtain that 


$$
\operatorname{lab}\left(\tilde{w}^{i, h}\right) \mathscr{R} e f
$$

Similarly, we have

$$
\operatorname{lab}\left({\widetilde{w^{\prime}}}^{i, h}\right) \mathscr{R} e \bar{f}
$$

for the idempotent $\bar{f}=\operatorname{lab}\left({\widetilde{w^{\prime}}}^{i, h} z_{h, i}\right)$. Here $f$ and $\bar{f}$ are $\mathscr{D}$-related by Lemma 4.6. Since $E \in \mathbf{L N B}$, we obtain by Result $1.5(\mathrm{i})$ that $e f=e \bar{f}$. Hence lab $\left(\tilde{w}^{i, h}\right) \mathscr{R}$ lab $\left({\widetilde{w^{\prime}}}^{i, h}\right)$ follows which implies also lab $\left(\tilde{w}^{g, h}\right) \mathscr{R}$ lab $\left(\widetilde{w}^{g, h}\right)$. Again utilizing Lemma 4.6, we infer by Result 1.7 the equality to be proved.

Lemma 4.10. Suppose that the F-cross-section $R$ in $S$ has the property that $\left(R^{\alpha}\right)$ is left normal and $\left(R^{\beta}\right)^{1}$ is right normal. If $\operatorname{ker} \sigma \in \mathbf{V}$ and $w, w^{\prime} \in A^{+}$such that $w \varrho(V, A) w^{\prime}$ then $\operatorname{lab}\left(\tilde{w}^{g, h}\right)=\operatorname{lab}\left({\widetilde{w^{\prime}}}^{g, h}\right)$ for every $g, h \in \operatorname{Obj}\left(T_{\mathrm{v}}(w)\right)$.

Proof. Since $\mathrm{S} \subseteq \mathrm{V}$, the assumptions imply $A(w)=A\left(w^{\prime}\right)$, and so $[w]=\left[w^{\prime}\right]$. Moreover, recall that $T_{\mathrm{v}}(w)=T_{\mathrm{v}}\left(w^{\prime}\right)$. It suffices to show that $\operatorname{lab}\left(\tilde{w}^{g, h}\right) \mathscr{R} \operatorname{lab}\left(\tilde{w}^{\prime g, h}\right)$. For, dually, we can see that they are also $\mathscr{L}$-related, and Lemma 4.6 ensures that they are $\gamma$-related. Hence the equality follows by Result 1.2 .

Consider the congruence relation $\zeta=\mathscr{R}_{S}^{\mathrm{b}} \cap \sigma$ on $S$. Clearly, $\zeta$ is idempotent pure.

If $(\varrho(\mathbf{V}))_{0} \nsubseteq h$ then $\operatorname{ker}(\sigma / \zeta) \in \mathbf{S}$ by Theorem 2.5 , that is, $S / \zeta$ is an inverse semigroup. On the other hand, $\zeta$ is idempotent pure and, since $\zeta \subseteq \mathscr{R}$, we see that $\zeta \mid E \subseteq \mathscr{D}_{E}$. Hence $\zeta=\gamma$. Thus Lemma 4.6 implies that $\operatorname{lab}\left(\tilde{w}^{g, h}\right) \zeta \operatorname{lab}\left({\widetilde{w^{\prime}}}^{\prime, h}\right)$ for every $g, h \in \operatorname{Obj}([w])$. This completes the proof in this case because $\zeta \subseteq \mathscr{R}$.

If $(\varrho(\mathbf{V}))_{0} \subseteq h$ and $(\varrho(\mathbf{V}))_{0} \nsubseteq \Delta$ then $\operatorname{ker}(\sigma / \zeta) \in \mathbf{L N B}$ by Theorem 2.5. Utilizing the remark before Lemma 4.5 , Lemma 4.9 ensures that $\operatorname{lab}\left(\tilde{w}^{g \cdot h}\right) \zeta \operatorname{lab}\left({\widetilde{w^{\prime}}}^{g, h}\right)$, completing the proof in this case, too.

Now assume that $(\varrho(V))_{0} \subseteq \Delta$. Then we have $\overline{0}(w)=\overline{0}\left(w^{\prime}\right)$ and, if $|A(w)|>1$, then also $0(w) \varrho\left(\mathbf{V}_{0}, A\right) 0\left(w^{\prime}\right)$ where $|A(0(w))|<|A(w)|$. We proceed by induction on $|A(w)|$. If $|A(w)|=1$ then one can easily check that the statement of the lemma holds. Suppose that the lemma is valid for any quasi- $F$-orthodox semigroup which possesses an $F$-crosssection $R$ such that $\left(R^{\alpha}\right)^{1}$ is left normal and $\left(R^{\beta}\right)^{1}$ is right normal, for any variety $\mathbf{V}$ with $\mathbf{S} \subseteq \mathbf{V}$ and for any $w, w^{\prime} \in A^{+}$with $|A(w)|<N(N>1)$. Consider $S, R, \mathbf{V}, w, w^{\prime}, g, h$ satisfying the assumptions of the lemma such that $|A(w)|=N$. Since $(\varrho(V))_{0} \subseteq \Delta$, we have $[0(w)]=\left[0\left(w^{\prime}\right)\right]$. Put $i=\omega(t(0(w))), j=\omega\left(t\left(0\left(w^{\prime}\right)\right)\right)$ and $k=\alpha(\overline{0}(w)), l=\omega(\overline{0}(w))$. Clearly, we have $i, j \in \operatorname{Obj}([0(w)])$ and $k, l \in \operatorname{Obj}([w])$. Since $(g, h) \in T_{\mathbf{v}}(w)$ and $(\varrho(V))_{0} \subseteq h$, we see that $g=\alpha(h(w))=\alpha(h(0(w)))$. Thus $(g, i),(g, j) \in T_{v_{0}}(0(w))=T_{v_{0}}\left(0\left(w^{\prime}\right)\right)$. Applying the induction hypothesis for $\hat{S}=S / \zeta, \mathbf{V}_{0}$ and $O(w) \bar{\varphi}, O\left(w^{\prime}\right) \bar{\varphi}$ (see that remark before Lemma 4.5), we infer that

$$
\operatorname{lab}\left(\widetilde{O(w)^{g . i}}\right) \zeta \operatorname{lab}\left({\widetilde{0\left(w^{\prime}\right.}}^{g \cdot i}\right)
$$

If $\left(\varrho\left(\mathbf{V}_{0}\right)\right)_{1} \subseteq t$ then we necessarily have $i=j$. Thus we obtain by multiplying (4.2) with $\operatorname{lab}\left(z_{i, k}\right)$ on the right that 


$$
\operatorname{lab}\left(\widetilde{0(w)^{g \cdot k}}\right) \zeta \operatorname{lab}\left({\widetilde{0\left(w^{\prime}\right.}}^{g \cdot k}\right)
$$

If $\left(\varrho\left(\mathbf{V}_{0}\right)\right)_{1} \nsubseteq t$ then $\mathbf{V}_{0} \subseteq \mathbf{L R B}$, and so, by Theorem 2.5 and Result $1.6, S / \zeta$ is $\mathscr{R}$-unipotent. The relation (4.2) implies

$$
\operatorname{lab}\left(\widetilde{0(w)}^{g, i}\right) \mathscr{R} \operatorname{lab}\left({\widetilde{0\left(w^{\prime}\right.}}^{g, j}\right)
$$

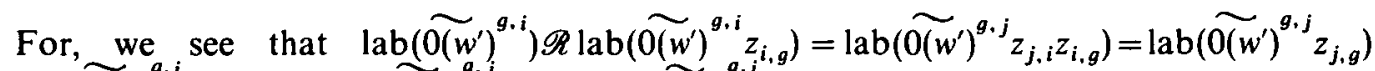
$\operatorname{lab}\left(\widetilde{0\left(w^{\prime}\right)^{g . i}} z_{i, g}\right)$ where $\widetilde{0\left(w^{\prime}\right)^{g . j}} z_{j, g}$ and $\widetilde{0\left(w^{\prime}\right)^{g, i} z_{i, g}}$ are $(g, g)$-paths in $\left[0\left(w^{\prime}\right)\right]$ which contain all the edges in $A\left(O\left(w^{\prime}\right)\right)$. Hence it follows by Lemma 4.6 that $\operatorname{lab}\left(\widetilde{0\left(w^{\prime}\right)}{ }_{g, j} z_{j, g}\right) \gamma \operatorname{lab}\left(\widetilde{O\left(w^{\prime}\right)} z_{i, g}\right)$. Since these are idempotents in $S$, we infer that

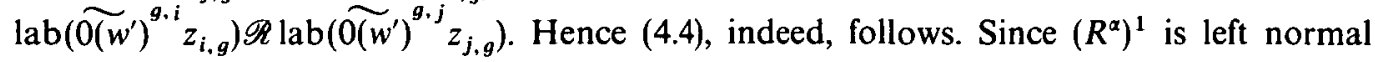
and $\zeta$ is an idempotent pure $\mathscr{R}$-unipotent congruence on $S$, Lemma 3.11 ensures that $\left(R^{\zeta}\right)^{1}$ is a left normal extended $F$-cross-section in $S / \zeta$. This implies by (4.4) that lab $\left(\widetilde{0(w)^{g, i}} z_{i, k}\right) \zeta \operatorname{lab}\left(\widetilde{0\left(w^{\prime}\right)^{g . j}} z_{j, k}\right)$, that is, (4.3) holds also in this case. However, we infer from (4.3) that

$$
\operatorname{lab}\left(\widetilde{l(w)^{g, l}}\right)=\operatorname{lab}\left(\widetilde{0(w)}^{g, k} \overline{0}(w)\right) \zeta \operatorname{lab}\left(\widetilde{0\left(w^{\prime}\right)}{ }^{g, k} \overline{0}\left(w^{\prime}\right)\right)=\operatorname{lab}\left({\widetilde{l\left(w^{\prime}\right.}}^{g, l}\right) .
$$

This relation implies by Lemma 4.7 and by $\zeta \subseteq \mathscr{R}$ that $\operatorname{lab}\left(\tilde{w}^{g, h}\right) \mathscr{R} \operatorname{lab}\left({\widetilde{w^{\prime}}}^{g, h}\right)$. The proof is complete.

Proof of Theorem 4.2. Let $(1, s)=w_{0}, w_{1}, \ldots, w_{n}=(1, t)$ be a sequence of words in $A^{+}$ such that $s, t \in S$ with $s \sigma t$ and, for every $i(i=0,1, \ldots, n-1), w_{i+1}$ is obtained from $w_{i}$ by one of the rules (S1), (S2) and (S2'). As we have mentioned at the beginning of this section, we have to prove that $s=t$. Put $g=s \sigma=t \sigma$. Consider the sequence $u_{-1}=$ $\left(1, s r_{g-1}\right), u_{0}=(1, s) z_{g, 1}=w_{0} z_{g, 1}, u_{1}=w_{1} z_{g, 1}, \ldots, u_{n}=w_{n} z_{g, 1}=(1, t) z_{g, 1}, u_{n+1}=\left(1, t r_{g^{-1}}\right)$. This sequence inherits the property that each word can be obtained from the previous one by one of the rules $(\mathrm{S} 1),(\mathrm{S} 2)$ and $\left(\mathrm{S} 2^{\prime}\right)$. Moreover, $1 \in \operatorname{Obj}\left(\left[u_{i}\right]\right)$ for $i=-1,0, \ldots, n+1$. In particular, if $(\varrho(\mathbf{V}))_{0} \subseteq h$ then $\alpha\left(h\left(u_{i}\right)\right)=1$ for every $i$. Thus $(1,1) \in T_{v}\left(u_{i}\right)$ for every $i$. Lemmas 4.8 and 4.10 imply that $\operatorname{lab}\left({\widetilde{u_{-1}}}^{1,1}\right)=\operatorname{lab}\left({\widetilde{u_{0}}}^{1,1}\right)=\ldots=\operatorname{lab}\left({\widetilde{u_{n}}}^{1,1}\right)=\operatorname{lab}\left({\widetilde{u_{n+1}}}^{1,1}\right)$. Since $\operatorname{lab}\left({\widetilde{u_{-1}}}^{1.1}\right)=s r_{g^{-1}}$ and $\operatorname{lab}\left({\widetilde{u_{n+1}}}^{1,1}\right)=t r_{g^{-1}}$, thus we infer that $s \mathscr{R}$. Dually, we obtain that $s \mathscr{L} t$ also holds. Since $S$ is $E$-unitary, the equality $s=t$ follows by Result 1.2 . This completes the proof of our theorem.

\section{REFERENCES}

1. C. C. Edwards, $F$-regular and F-orthodox semigroups, Semigroup Forum 19 (1980), 331-345.

2. S. Eilengerg, Automata, Languages and Machines, Vol. B (Academic Press, New York, 1976).

3. T. E. HALL, Congruences and Green's relations on regular semigroups, Glasgow Math. J. 13 (1972), 167-175.

4. J. M. Howie, An Introduction to Semigroup Theory (Academic Press, London, 1976). 
5. J. KaDourek and M. B. Szendrei, A new approach in the theory of orthodox semigroups, Semigroup Forum 40 (1990), 257-296.

6. K. S. S. Nambooripad, The natural partial order on a regular semigroup, Proc. Edinburgh Math. Soc. 23 (1980), 249-260.

7. L. O'CARroll, Embedding theorems for proper inverse semigroups, J. Algebra 42 (1976), $26-40$.

8. F. PAStun, The lattice of completely regular semigroup varieties, preprint.

9. M. Petrich, A construction and a classification of bands, Math. Nachr. 48 (1971), 263-274.

10. M. Petrich, Inverse semigroups (Wiley \& Sons, New York, 1984).

11. L. Polák, On varieties of completely regular semigroups I, Semigroup Forum 32 (1985), 97-123.

12. L. Polák, On varieties of completely regular semigroups II, Semigroup Forum 36 (1987), 253-284.

13. M. B. Szendrel, E-unitary regular semigroups, Proc. Roy. Soc. Edinburgh 106A (1987), 89-102.

14. M. B. Szendrei, On E-unitary covers of orthodox semigroups, Internat. J. Algebra Comput. 3 (1993), 317-333.

15. M. B. Szendrei, On embeddability into a semidirect product of an orthodox semigroup by a group, Acta Sci. Math. 57 (1993), 601-612.

16. K. TakizaWa, E-unitary $\mathscr{R}$-unipotent semigroups, Bull. Tokyo Gakugei Univ. (4) 30 (1978), 21-33.

17. B. Tilson, Categories as algebra: an essential ingredient in the theory of monoids, $J$. Pure Appl. Algebra 48 (1987), 83-198.

18. P. S. Venkatesan, Right (left) inverse semigroups, J. Algebra 31 (1974), 209-217.

Fachbereich Mathematik

Gesamthochschule Kassel

Holländische Str. 36

D-W-34109 KASSEL

Germany
Bolyal INSTITUTE József AtTila University Aradi Vertanúk Tere 1 H-6720 SzEGED

Hungary 